\title{
COMPORTAMIENTO SÍSMICO DE EDIFICIOS CON BASE EN MARCOS DÚCTILES DE CONCRETO REFORZADO CON CONTRAVENTEO CHEVRÓN
}

\author{
Eber Alberto Godínez Domínguez, ${ }^{(1)}$, Arturo Tena Colunga ${ }^{(2)}$ y Luis Eduardo Pérez Rocha ${ }^{(3)}$
}

\begin{abstract}
RESUMEN
Se presenta un estudio en que se evalúa, mediante análisis dinámicos no lineales paso a paso, el comportamiento sísmico de edificios de diferentes alturas (ocho, 15 y 24 niveles) estructurados con base en marcos dúctiles de concreto reforzado con contraventeo metálico tipo chevrón. Los edificios se diseñaron para tres diferentes zonas (zona costa del estado de Guerrero y zonas IIIa y IIIb del lago del Distrito Federal), de acuerdo a la altura de cada modelo. De los resultados expuestos, es posible concluir que si se emplean conceptos de diseño por capacidad, así como parámetros de diseño específicos al sistema estructural considerado, es posible obtener capacidades de deformación y sobrerresistencia adecuados, así como un desempeño estructural satisfactorio.
\end{abstract}

Palabras Clave: marcos dúctiles contraventeados; contraventeo chevrón; marcos de concreto reforzado; diseño por capacidad; análisis dinámicos no lineales; desempeño estructural

\begin{abstract}
In this paper the authors summarize the results of a study devoted to evaluate, using nonlinear dynamic analyses, the seismic behavior of ductile moment-resisting reinforced concrete concentric braced frames structures (RC-MRCBFs) using chevron steel bracing (eight, 15 and 24 stories in height). RC-MRCBFs were assumed to be located in three different soil conditions in Mexico (coastline zone of the state of Guerrero and lakebed zones IIIa and IIIb of Mexico City). From the results obtained, it is possible to conclude that if capacity design principles and specific design parameters for the new design of RC-MRCBFs are used, suitable global ductility capacities and overstrength demands are obtained, and a satisfactory structural performance is achieved.
\end{abstract}

Key Words: ductile braced frames; chevron bracing; reinforced concrete frames; capacity design; nonlinear dynamic analysis; structural performance.

Artículo recibido el 10 de diciembre de 2010 y aprobado para su publicación el 5 de diciembre de 2011.

(1) Facultad de Ingeniería, Universidad Autónoma de Chiapas, Campus-I, Blvd. Belisario Domínguez, kilómetro 1081, Sin número, Col. Terán, 29050, Tuxtla Gutiérrez, Chiapas, México, e-mail: eber.godinez@unach.mx

(2) Departamento de Materiales, Universidad Autónoma Metropolitana Azcapotzalco, Av. San Pablo 180, Col. Reynosa Tamaulipas, 02200 México, DF, e-mail: atc@ correo.azc.uam.mx

(3) Instituto de Investigaciones Eléctricas, Calle Reforma 113, Col. Palmira, 62490, Cuernavaca, Morelos, e-mail: lepr@iie.org.mx 


\section{INTRODUCCIÓN}

En años recientes el empleo de marcos de concreto reforzado contraventeados se ha estudiado no únicamente desde un enfoque de reparación, si no desde el punto de vista de diseño de edificaciones nuevas. Algunos investigadores se han enfocado en el estudio del comportamiento sísmico y el desarrollo de parámetros de diseño para diferentes configuraciones de contraventeo (Maheri y Sahebi 1997, Maheri y Akbari 2003, Maheri y Hadjipour 2003, Youssef et al. 2007, Godínez-Domínguez y Tena-Colunga 2008, Maheri y Ghaffarzadeh 2008, Godínez y Tena 2009 y 2011, Godínez-Domínguez y Tena-Colunga 2010 y Godínez 2010). A continuación se presenta un resumen de los trabajos desarrollados por los autores de referencia:

Maheri y Sahebi (1997) realizaron uno de los primeros estudios experimentales enfocados en la determinación de la eficiencia de diferentes arreglos de contraventeo metálico para incrementar la resistencia a cortante de marcos planos de concreto reforzado como sistema estructural para el diseño de estructuras nuevas. De las pruebas experimentales observaron un incremento significativo de la resistencia del marco de concreto. Comentan que si se emplea un esquema de conexión adecuado entre el sistema de contraventeo y el marco de concreto, esta puede ser una alternativa o complemento viable a los muros de cortante en el diseño ante cargas laterales de edificios de concreto reforzado.

Posteriormente, Maheri y Akbari (2003) estudiaron marcos de concreto reforzado con ductilidad intermedia con contraventeo metálico con configuraciones en $\mathrm{X}$ y $\mathrm{K}$ de diferentes alturas. Asimismo, Maheri et al. (2003) estudiaron, de manera complementaria, el mismo caso pero para marcos de un nivel y una crujía con la finalidad de realizar calibraciones para el análisis de modelos más complejos. Ellos propusieron, con base en los resultados de análisis estáticos no lineales ante carga monótona creciente, factores de comportamiento sísmico $\left(R_{w}\right)$ para diferentes niveles de demandas de ductilidad. Observaron que existe una dependencia de los factores $R_{w}$ tanto con base en el porcentaje de fuerza cortante que resiste el sistema de contraventeo, el número de niveles y el esquema de contraventeo empleado. Consideran que en los marcos con contraventeo en $\mathrm{K}$ es recomendable diseñar el sistema de contraventeo para resistir el mayor porcentaje de carga lateral; sin embargo, para el caso de los modelos con contraventeo en X resulta mas eficiente distribuir uniformemente la fuerza lateral entre el marco y los contravientos.

Maheri y Hadjipour (2003), mediante un programa experimental, estudiaron el comportamiento de tres esquemas de conexión de sistemas de contraventeo metálico a marcos de concreto reforzado, con la finalidad de realizar recomendaciones para el diseño de conexiones de estructuras nuevas con base en marcos de concreto reforzado con contraventeo de acero. La técnica empleada para realizar las conexiones consiste en fijar una placa de acero a los miembros de concreto a la cual posteriormente se suelda una placa de conexión encargada de recibir los contravientos metálicos. Con base en las pruebas experimentales de las conexiones a escala real llevadas a cabo en una máquina universal, comentan que las conexiones pueden diseñarse satisfactoriamente siguiendo simultáneamente las recomendaciones existentes para diseño de elementos de acero y concreto (en este caso las recomendaciones del LRFD-94 y el ACI-318-95, respectivamente).

Ghaffarzadeh y Maheri (2006) y Youssef et al. (2007), mediante pruebas experimentales cíclicas de subestructuras a escala, así como de estudios analíticos, evaluaron el comportamiento tanto de marcos de concreto reforzado con contraventeo metálico, como de marcos no contraventeados, comparando las ventajas y desventajas de cada caso. Los dos estudios citados son muy similares; de hecho, la primera parte de ambos podría considerarse como la misma. En ambos casos se destacan, mayoritariamente, las ventajas de los sistemas contraventeados respecto a los marcos momento-resistentes. Los autores evalúan parámetros como la degradación de rigidez, la ductilidad y la disipación de energía. Ambos estudios destacan el buen comportamiento de los marcos de concreto reforzado contraventeados, los cuales al 
igual que en el estudio desarrollado por Maheri y Hadjipour (2003), fueron diseñados como sistemas de ductilidad intermedia.

Maheri y Ghaffarzadeh (2008), realizaron un proyecto experimental y analítico en marcos de concreto reforzado con contraventeo en cruz (X), en que proponen una expresión para la determinación del nivel de sobrerresistencia por efecto de las conexiones en función de factores que dependen del número de crujías contraventeadas y el número de niveles del marco en estudio, así como de un índice que estima la sobrerresistencia obtenida con base en un modelo de cuatro niveles a partir de la relación entre la rigidez efectiva de un marco con conexiones del sistema de contraventeo al marco de concreto y la rigidez efectiva de un marco sin conexiones de este tipo. Los autores consideran que dicha sobrerresistencia es significativa y debe considerarse en el diseño.

Los detalles de cada uno de los estudios comentados se encuentran en las referencias citadas.

Este estudio representa la segunda etapa de una investigación integral, enfocada tanto a la obtención de parámetros específicos para el diseño de edificios con base en marcos dúctiles de concreto reforzado con contraventeo tipo chevrón, como en la evaluación de una metodología de diseño por capacidad adaptada de los lineamientos de las Normas Técnicas Complementarias del Reglamento de Construcciones para el Distrito Federal vigente. En Godínez y Tena (2009 y 2010), Godínez-Domínguez y Tena-Colunga (2010) y Godínez (2010) se presentan algunos parámetros que se consideran útiles para el diseño de estructuras nuevas con base el sistema estructural comentado. Los parámetros de diseño se obtuvieron a partir de los resultados de análisis estáticos no lineales ante carga monótona creciente de modelos de 4, 8, 12, 16, 20 y 24 niveles. En el presente artículo se emplean las recomendaciones de diseño derivadas de los estudios comentados para el diseño de edificios con base en marcos dúctiles de concreto reforzado con contraventeo metálico chevrón de diferentes alturas, localizados en diferentes zonas sísmicas. El comportamiento sísmico de cada diseño se evaluó mediante análisis dinámicos no lineales paso a paso, empleando para este fin registros acelerométricos sintéticos que representan el peligro sísmico considerado en los códigos de diseño usados.

El objetivo de este estudio es evaluar el comportamiento sísmico de edificios con base en marcos dúctiles de concreto reforzado con contraventeo chevrón diseñados con base en una metodología de diseño por capacidad adaptada de los lineamientos de las Normas Técnicas Complementarias del Reglamento de Construcciones para el Distrito Federal vigente, y empleando parámetros de diseño obtenidos en los trabajos previos citados. Lo anterior, con la finalidad de valorar la factibilidad del uso seguro de este sistema estructural para la construcción de edificaciones nuevas empleando factores de comportamiento sísmico altos $(Q=4)$ en los que se obtengan mecanismos cercanos al supuesto de columna fuerte - viga débil - contraviento más débil. Asimismo, se comparan algunos de los parámetros de diseño obtenidos previamente con base en análisis ante carga monótona creciente, contra los obtenidos con base en las respuestas promedio derivadas de los análisis dinámicos no lineales paso a paso, dentro de los que figuran: factores de reducción por sobrerresistencia $(R)$, distorsiones de entrepiso asociadas a los estados límite de servicio, distorsiones de entrepiso asociadas al estado límite de prevención de colapso.

\section{EDIFICIOS ESTUDIADOS}

Se describen a continuación las características principales de seis edificios diseñados conforme a dos distintos reglamentos: el Manual de Obras Civiles de la Comisión Federal de Electricidad (MOC2008) y el Reglamento de Construcciones del Distrito Federal (RCDF-04). Los edificios cuentan con ocho, 15 y 24 niveles. En todos los casos se empleó un factor de comportamiento sísmico $Q=4$. 


\section{Descripción de los edificios y modelos de análisis}

Se diseñaron seis edificios para albergar oficinas de ocho, 15 y 24 niveles, dos para cada altura en estudio. Los edificios de ocho y 15 niveles son regulares tanto en planta como en elevación. Sin embargo, los edificios de 24 niveles son irregulares, pues su relación altura a base menor excede el valor de 2.5 especificado en las Normas Técnicas Complementarias para Diseño por Sismo (NTCS-04) para ser considerada como regular, por lo que son irregulares por esbeltez. El primer tipo de edificio (T1) cuenta con una planta rectangular de $21 \mathrm{~m}$ de ancho (dirección $Y$ ó N-S) y $32 \mathrm{~m}$ de largo (dirección $X$ ó E-W), en la que cada marco perimetral se encuentra contraventeado en sus crujías exteriores. El edificio cuenta con cuatro crujías en la dirección $X$ y tres en la dirección $Y$ con claros de 8 y 7 metros, respectivamente (figura 1a). La configuración estructural seleccionada ha sido empleada en estudios previos de marcos dúctiles de concreto reforzado (Luaces 1995, Tena et al. 1997, Luna 2000, Correa 2005, Gatica 2007, Tena y Correa 2008, Tena-Colunga et al. 2008) y edificios con primer piso suave (Tena-Colunga 2010). Existen vigas secundarias orientadas en ambas direcciones como en dos estudios previos (Gatica 2007, Tena-Colunga $e t$ al. 2008). La altura de entrepiso es de $3.40 \mathrm{~m}$, constante para los modelos ocho, $15 \mathrm{y} 24$ niveles, por lo que la altura total de cada edificio es de $27.2 \mathrm{~m}, 51.0 \mathrm{~m}$ y $81.6 \mathrm{~m}$, respectivamente (figura 1).

El segundo tipo de edificio (T2) cuenta con una planta rectangular de $21 \mathrm{~m}$ de ancho (dirección $Y$ ó $\mathrm{N}-\mathrm{S}$ ) y $35 \mathrm{~m}$ de largo (dirección $X$ ó E-W). A diferencia de los edificios tipo 1 (ET1), en este caso, se encuentran contraventeadas las crujías interiores y exteriores tanto en marcos perimetrales, como en marcos interiores (figura 2). El edificio cuenta con cinco crujías en la dirección $X$ y tres en la dirección $Y$, con claros de 7 metros. Existen vigas secundarias orientadas en ambas direcciones (figura 2a). La altura de entrepiso es la misma que en los edificios tipo uno $(3.4 \mathrm{~m})$, por lo que las alturas de los edificios diseñados son las mismas que en los casos anteriores.

En adelante, se emplearan los sufijos T1 y T2 para hacer referencia a los edificios tipo 1 у 2 , respectivamente; por ejemplo, la nomenclatura $15 \mathrm{~T} 2$ hará referencia a un edificio de 15 niveles cuya planta y elevaciones corresponden a las mostradas en la figura 2.

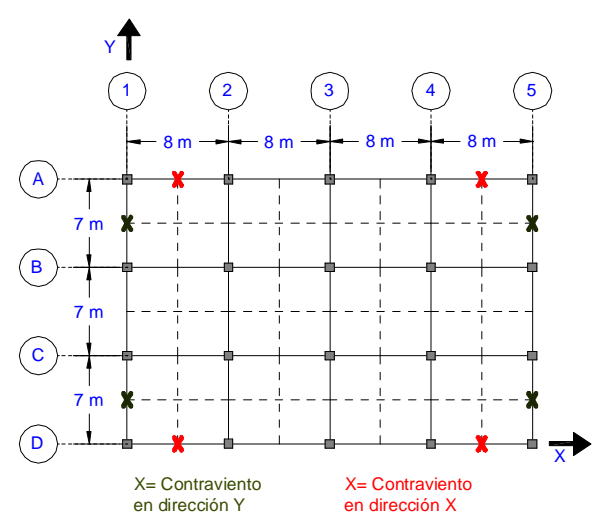

a) Planta tipo
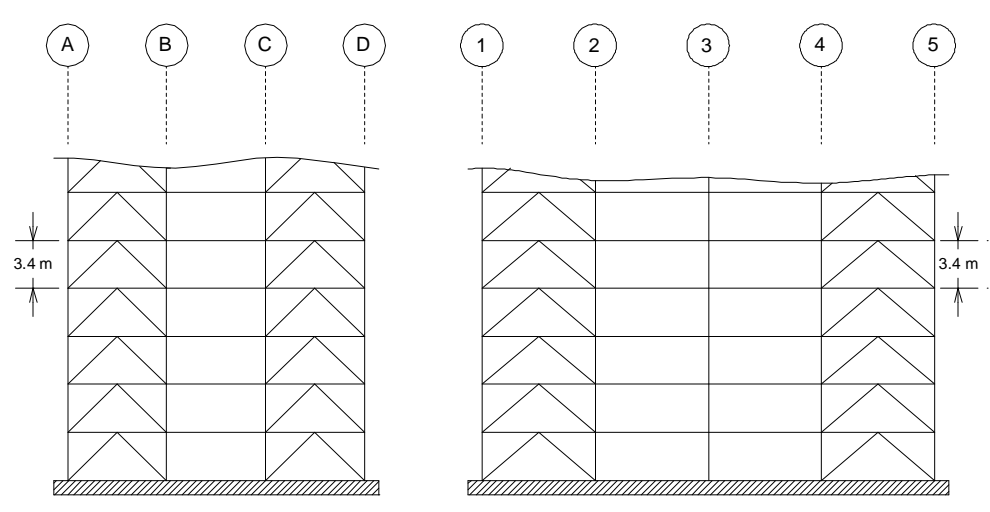

b) Elevación de los marcos perimetrales, dirección $Y$ (ejes 1 y 5) y dirección $X$ (ejes A y D)

Figura 1. Planta y elevaciones de los edificios tipo 1 (T1) 


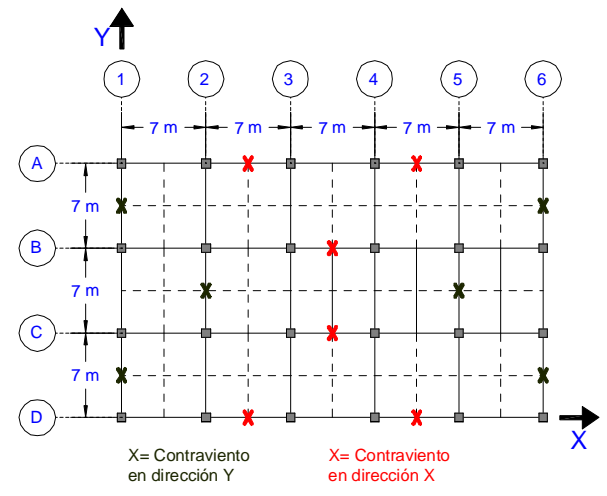

a) Planta tipo
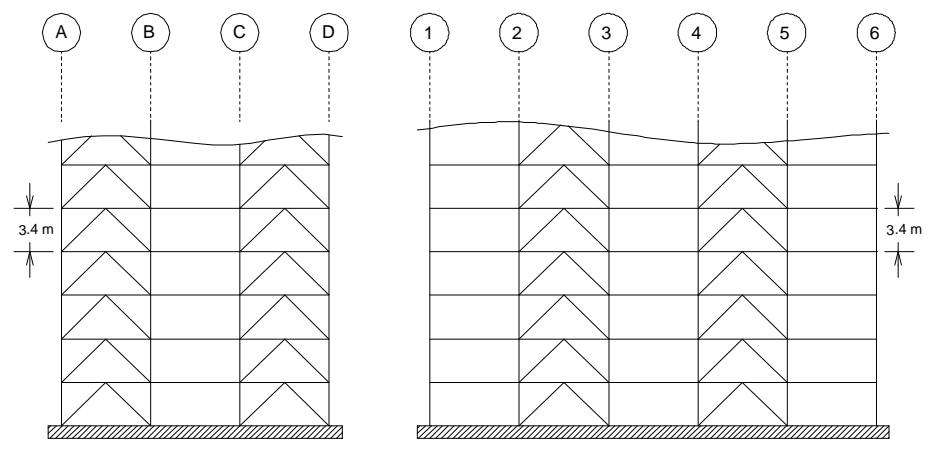

b) Elevación de los marcos perimetrales, dirección $Y$ (ejes 1 y 6 ) y dirección $X$ (ejes A y D)

Figura 2. Planta y elevaciones de los edificios tipo 2 (T2)

\section{METODOLOGÍA DE DISEÑO}

Para el diseño de los elementos sismorresistentes de cada uno de los edificios considerados, se empleó una metodología basada en conceptos por capacidad, donde los elementos estructurales se diseñan por resistencia del más débil al más fuerte, por lo que para obtener un mecanismo de colapso columna fuerte - viga débil - contraviento más débil, la secuencia de diseño debe ser: 1) contravientos, 2) vigas, 3) columnas y, 4) conexiones. Asimismo, se emplearon las recomendaciones de diseño derivadas de una serie de análisis estáticos no lineales ante carga monótona creciente de marcos planos de entre cuatro y 24 niveles, los cuales se describen con detalle en Godínez y Tena (2009 y 2011), Godínez-Domínguez y Tena-Colunga (2008 y 2010) y Godínez (2010). Por lo anterior, para el cálculo de los factores de reducción por sobrerresistencia $(R$, ec. 1$)$, los límites de distorsión asociados al estado límite de servicio (ec. 2) y prevención de colapso (ecs. 3 y 4, en este caso se consideró también la propuesta de las NTCS04), así como los balances de resistencia (ec. 5) y consideraciones para el diseño a nivel local, se emplearon las recomendaciones derivadas de la primera parte de este estudio, reportada en los estudios citados.

$$
R=\left\{\begin{array}{lr}
1.7+2.3\left(1-\sqrt{T_{e} / T_{a}}\right) & \text { si } T_{e} \leq T_{a} \\
1.7 ; & \text { si } T_{e}>T_{a}
\end{array}\right.
$$

$\Delta_{y}=0.002$

$\Delta_{\text {max -propuesto }}=0.013$

$\Delta_{\max -\mathrm{NTCS}-04}=0.015$

$V_{R C o l} \geq 50+1.2\left(\frac{H}{L}\right)^{2}$ 
donde $T_{a}$ y $T_{e}$ son el periodo característico que define el inicio de la meseta en el espectro de diseño elástico y el periodo fundamental elástico de la estructura, $\Delta_{y}$ es la distorsión de fluencia considerada en la revisión estado límite de servicio, $\Delta_{\max }$ es la distorsión máxima de diseño considerada en la revisión del estado límite de colapso, $V_{R C o l}$ representa la aportación mínima de las columnas al cortante resistente ante carga lateral (en porcentaje), $H$ es la altura total del edificio y $L$ la longitud de la base de la estructura en el sentido de análisis.

El criterio empleado para la definición del porcentaje de la resistencia a fuerza cortante total que el sistema de contraventeo debe ser capaz de aportar, se hizo con base en la propuesta de Godínez y Tena (2009) en donde se presenta una ecuación simple en función de la relación de esbeltez de la estructura $(H / L)$, para la estimación del porcentaje mínimo de resistencia a fuerza cortante que las columnas de un entrepiso deben ser capaces de aportar en el sistema dual (ecuación 5). Con lo anterior se busca que los mecanismos de colapso obtenidos sean congruentes con la filosofía de diseño, es decir, columnas fuerteviga débil-contraviento más débil. La propuesta es congruente con la recomendación de las NTCS-04, en que se especifica que para el diseño de estructuras dúctiles en que la resistencia en todos los entrepisos es suministrada por marcos contraventeados, se requiere que en cada entrepiso los marcos sean capaces de resistir, sin contar con contravientos, cuando menos 50 por ciento de la fuerza sísmica actuante. Debe notarse que la propuesta de las NTCS-04 es independientemente de la relación de esbeltez de la estructura considerada.

Los modelos para el análisis estructural se elaboraron con el programa ETABS en su versión 9.0.0 (ETABS 2005). Para realizar el diseño de la estructura se llevó a cabo un análisis modal espectral que incluyó todos los modos que garantizaran que la suma de los pesos efectivos en cada dirección de análisis sea mayor o igual a 90 por ciento del peso total de la estructura. Se incluyeron los efectos P- $\Delta$ en los análisis. Por conveniencia en la interpretación de resultados, se desprecian los efectos de interacción suelo-estructura.

Las particularidades de cada diseño, incluidas las dimensiones de vigas y columnas y sus respectivos refuerzos por flexión y cortante, así como secciones de contravientos, relaciones de esbeltez y su variación en altura, se reportan con detalle en Godínez (2010).

\section{Consideraciones para el análisis estructural}

Para la elaboración de los modelos de análisis estructural se consideró el aporte de la losa en rigidez, es decir, al modelar las vigas, éstas se consideraron como vigas de sección $L$ en el caso de los marcos perimetrales y vigas de sección $T$ para los marcos interiores. El espesor de los patines de cada elemento se obtuvo mediante el diseño de las losas, las cuales fueron previamente diseñadas, como es costumbre, para tomar cargas verticales y satisfacer estados límites de deformación y de servicio. Asimismo, las vigas secundarias se diseñaron bajo el efecto de cargas verticales, revisando el cumplimiento del estado limite de servicio tal y como se indica en el apartado 3.2 de las NTCC-04.

Con la finalidad de tener una idea razonable de las propiedades dinámicas de la estructura, y a su vez evaluar la variación en las demandas sísmicas, se realizaron dos modelos de análisis (para un modelo de ocho y un modelo de 15 niveles). En el primer modelo no se toma en cuenta la disposición de la sección 1.3.1 de las NTCC-04 referente a considerar secciones agrietadas en la elaboración de los modelos de análisis estructural, es decir, se consideraron secciones gruesas. Por otra parte, en el segundo modelo de análisis si se considera el agrietamiento de las vigas mediante la reducción de su momento de inercia en $50 \%$. En ambos modelos se consideró que la rigidez en los nudos es $50 \%$ de la que tendrían si fueran 
infinitamente rígidos a flexión, tal y como es comúnmente considerado por los despachos de cálculo estructural y como lo recomiendan Horvillieur y Cheema (1994) y Tena (2007).

Para ambos modelos de análisis se calcularon las distorsiones de entrepiso y se compararon con las máximas permisibles. Asimismo, en cada caso se determinó el refuerzo por flexión necesario de las vigas, que de acuerdo a la metodología planteada, se diseñan con base en los elementos mecánicos obtenidos directamente del análisis estructural. Finalmente, los suministros de refuerzo por flexión en vigas quedaron definidos de forma que se satisficieran simultáneamente las demandas de ambos modelos de análisis.

Respecto a lo anterior, debe considerarse que para que las vigas de estos sistemas duales presentaran niveles de agrietamiento tan elevados como los considerados en la sección 1.3.1 de las NTCC04, debería presentarse previamente el pandeo o desconexión de los elementos de contraventeo. Lo anterior indica que al considerar dichos niveles de agrietamiento en las vigas del edificio, también debería considerarse que no existe ya la línea de defensa provista por el sistema de contraventeo.

\section{Espectros para diseño sísmico}

El criterio empleado para la ubicación geográfica de cada edificio se basa en tratar que los periodos dominantes del terreno fueran lo más próximos posible a los periodos fundamentales de los edificios, es decir, que el periodo fundamental de cada modelo (en cada dirección), coincida con la zona de mayores demandas de aceleración especificadas por los espectros de diseño correspondientes (figuras 4, 6 y 7).

\section{Edificios de ocho niveles}

Dadas las características dinámicas de los edificios de ocho niveles (tabla 1), estos fueron ubicados para su diseño en la costa de Guerrero, una de las zonas en que, de acuerdo al Manual de Obras Civiles de la Comisión Federal de Electricidad (MOC-2008), se presentan las máximas demandas de aceleración en la República Mexicana (figura 3). En la tabla 1 se muestra también para cada edificio el porcentaje de masa asociada a cada modo.

Para la definición del espectro elástico se tomaron en cuenta las recomendaciones del MOC-2008 (2009), según lo comentado con detalle en Tena-Colunga et al. (2009). Para la definición del espectro de diseño (espectro inelástico) asociado al estado límite de prevención de colapso, de acuerdo a lo estipulado en el MOC-2008, las ordenadas espectrales de aceleración asociadas al espectro de diseño elástico se redujeron por concepto de ductilidad $\left(Q^{\prime}\right)$, sobrerresistencia $(R)$ y redundancia $(\rho)$.

El factor de reducción de fuerzas sísmicas $Q$ ' se calculó de acuerdo con lo especificado en el MOC2008. Por otro lado, para el cálculo de los factores de reducción por sobrerresistencia $(R)$, al igual que para los edificios de 15 y 24 niveles, se emplearon los valores propuestos en la ecuación 1, los cuales como se comentó, se definieron con base en estudios analíticos y representan los niveles de sobrerresistencia particulares para el sistema estructural es estudio (Godínez 2010).

Como se comenta en el MOC-2008 y en Tena-Colunga et al. (2009), el espectro de aceleraciones para realizar la revisión del estado límite de servicio se obtuvo indirectamente dividiendo las ordenadas del espectro definido para la revisión del estado límite de prevención de colapso por una factor igual a 5.5 y considerando un comportamiento lineal del suelo. 
Tabla 1. Características dinámicas de los edificios 8T1 y 8T2

\begin{tabular}{|c|c|c|c|c|c|c|c|}
\hline \multirow{3}{*}{ Modo } & \multirow{3}{*}{ Dirección } & \multicolumn{3}{|c|}{ Edificio $8 T 1$} & \multicolumn{3}{|c|}{ Edificio $8 T 2$} \\
\hline & & \multirow{2}{*}{$\begin{array}{c}\text { Periodo } \\
(s)\end{array}$} & \multicolumn{2}{|c|}{$\begin{array}{c}\text { Masa Modal } \\
(\%)\end{array}$} & \multirow{2}{*}{$\begin{array}{c}\text { Periodo } \\
\text { (s) }\end{array}$} & \multicolumn{2}{|c|}{$\begin{array}{c}\text { Masa Modal } \\
(\%)\end{array}$} \\
\hline & & & $E-W$ & $N-S$ & & $E-W$ & $N-S$ \\
\hline 1. Primer modo traslación & E-W & 0.554 & 76.72 & 0.00 & 0.5284 & 77.47 & 0.00 \\
\hline 2. Primer modo traslación & $\mathrm{N}-\mathrm{S}$ & 0.542 & 0.00 & 77.86 & 0.5007 & 0.00 & 77.52 \\
\hline 3. Primer modo de rotación & & 0.421 & 0.00 & 0.00 & 0.4137 & 0.00 & 0.00 \\
\hline 4. Segundo modo traslación & E-W & 0.184 & 12.52 & 0.00 & 0.1767 & 12.13 & 0.00 \\
\hline 5. Segundo modo traslación & $\mathrm{N}-\mathrm{S}$ & 0.174 & 0.00 & 11.82 & 0.1670 & 0.00 & 12.43 \\
\hline 6. Segundo modo de rotación & & 0.140 & 0.00 & 0.00 & 0.1402 & 0.00 & 0.00 \\
\hline 7. Tercer modo traslación & E-W & 0.098 & 4.68 & 0.00 & 0.0960 & 4.53 & 0.00 \\
\hline 8. Tercer modo traslación & $\mathrm{N}-\mathrm{S}$ & 0.094 & 0.00 & 4.53 & 0.0909 & 0.00 & 4.39 \\
\hline 9. Tercer modo de rotación & & 0.078 & 0.00 & 0.00 & 0.0777 & 0.00 & 0.00 \\
\hline$\Sigma \%$ masas modales & & & 93.91 & 94.21 & & 94.13 & 94.35 \\
\hline
\end{tabular}

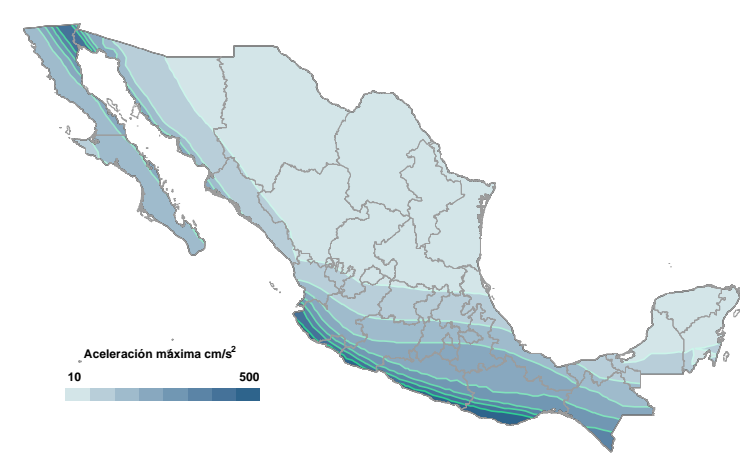

a) Aceleraciones máximas en roca $a_{0}^{r}$

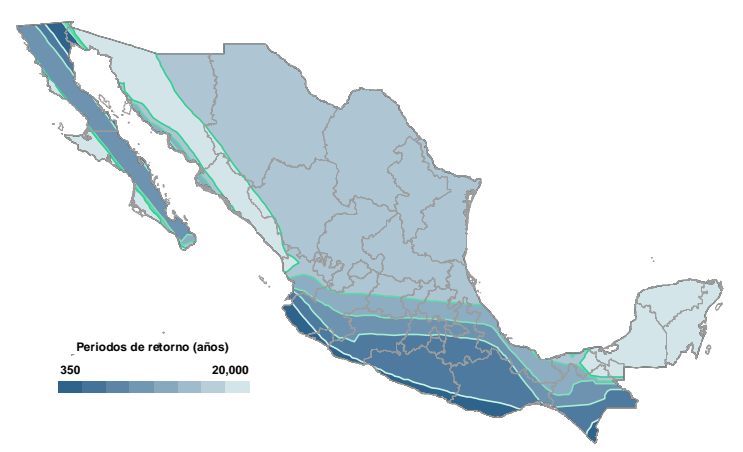

b) Periodos de retorno asociados, $T_{r}$ (años)

Figura 3. Aceleraciones máximas del terreno acorde al MOC-2008 asociadas a periodos de retorno obtenidos empleando criterios de diseño óptimo (MOC-2008, 2009, Tena-Colunga et al. 2009)

En la figura 4 se muestra el espectro de diseño elástico correspondiente a terreno firme (roca) de acuerdo al MOC-2008 (2009), empleado para el diseño de los modelos de ocho niveles. Asimismo, se muestra el espectro inelástico de diseño, y el asociado al estado límite de servicio. Las líneas verticales indican el intervalo de periodos en que se encuentran ubicados los modelos de ocho niveles.

\section{Edificios de 15 niveles}

Los edificios de 15 niveles (15T1 y 15T2) se ubicaron para su diseño en la zona III $_{\mathrm{a}}$ conforme a la zonificación sísmica de las NTCS-04, pues a ésta corresponden periodos dominantes del terreno cercanos a los periodos fundamentales de estos edificios (tabla 2). El someter a la estructura a una serie de análisis dinámicos, empleando registros acelerométricos asociados a esta zona (con periodos cercanos a los dominantes de los edificios), favorece a inducir en la estructura demandas de comportamiento inelástico importantes.

Para la definición del espectro de diseño elástico, conforme a lo especificado en el Apéndice A de las NTCS-04 (AA), se consideró como periodo dominante más largo del terreno un valor $T_{s}=1.2$ segundos. Se obtuvieron además los siguientes parámetros: (a) coeficiente de aceleración del terreno $a_{0}=$ 
0.205, (b) coeficiente sísmico $c=0.924$, (c) $k=0.80 \mathrm{y}$, (d) los periodos característicos $T_{a}=0.655 \mathrm{~s}$ y $T_{b}=$ 1.44 s. Dado que no se consideró la interacción suelo-estructura, el factor de reducción por amortiguamiento suplementario se considero unitario $(\beta=1)$.

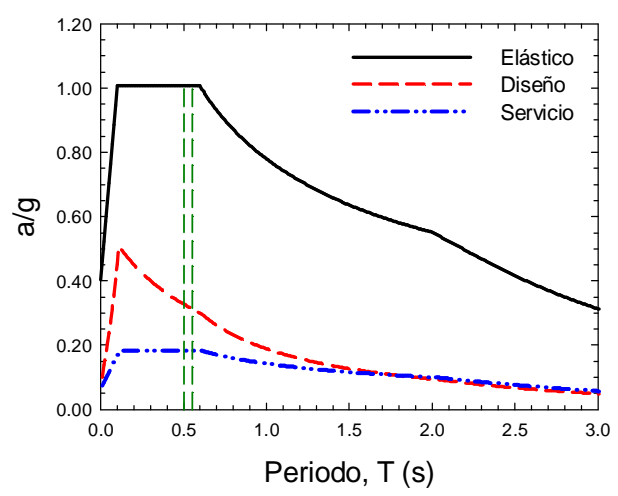

Figura 4. Espectro de diseño elástico, reducido por ductilidad y sobrerresistencia y de servicio en roca de acuerdo al MOC-2008

Tabla 2. Características dinámicas de los edificios $15 \mathrm{~T} 1$ y $15 \mathrm{~T} 2$

\begin{tabular}{|c|c|c|c|c|c|c|c|}
\hline \multirow{3}{*}{ Modo } & \multirow{3}{*}{ Dirección } & \multicolumn{3}{|c|}{ Edificio $15 T 1$} & \multicolumn{3}{|c|}{ Edificio $15 T 2$} \\
\hline & & \multirow{2}{*}{$\begin{array}{c}\text { Periodo } \\
\text { (s) }\end{array}$} & \multicolumn{2}{|c|}{$\begin{array}{c}\text { Masa Modal } \\
(\%)\end{array}$} & \multirow{2}{*}{$\begin{array}{l}\text { Periodo } \\
\text { (s) }\end{array}$} & \multicolumn{2}{|c|}{$\begin{array}{c}\text { Masa Modal } \\
(\%)\end{array}$} \\
\hline & & & $E-W$ & $N-S$ & & $E-W$ & $N-S$ \\
\hline 1. Primer modo traslación & E-W & 1.212 & 73.49 & 0.00 & 1.060 & 76.17 & 0.00 \\
\hline 2. Primer modo traslación & $\mathrm{N}-\mathrm{S}$ & 1.143 & 0.00 & 74.31 & 1.061 & 0.00 & 73.81 \\
\hline 3. Primer modo de rotación & & 0.860 & 0.00 & 0.00 & 0.840 & 0.00 & 0.01 \\
\hline 4. Segundo modo traslación & E-W & 0.414 & 13.34 & 0.00 & 0.363 & 12.05 & 0.00 \\
\hline 5. Segundo modo traslación & $\mathrm{N}-\mathrm{S}$ & 0.390 & 0.00 & 12.96 & 0.358 & 0.00 & 13.71 \\
\hline 6. Segundo modo de rotación & & 0.302 & 0.00 & 0.00 & 0.291 & 0.00 & 0.01 \\
\hline 7. Tercer modo traslación & E-W & 0.232 & 4.69 & 0.00 & 0.209 & 4.24 & 0.00 \\
\hline 8. Tercer modo traslación & $\mathrm{N}-\mathrm{S}$ & 0.215 & 0.00 & 4.58 & 0.203 & 0.00 & 4.68 \\
\hline 9. Tercer modo de rotación & & 0.172 & 0.00 & 0.00 & 0.169 & 0.00 & 0.00 \\
\hline$\Sigma \%$ masas modales & & & 91.52 & 91.85 & & 92.46 & 92.20 \\
\hline
\end{tabular}

Para la definición del espectro inelástico de diseño, de acuerdo a lo especificado en el AA, las ordenadas espectrales de aceleración asociadas al espectro de diseño elástico se redujeron por concepto de ductilidad $\left(Q^{\prime}\right)$ y sobrerresistencia $(R)$. Para realizar la reducción por ductilidad se emplearon las ecuaciones propuestas en el AA. Por otro lado, para el cálculo de los factores de reducción por sobrerresistencia $(R)$, al igual que para los edificios de ocho niveles, se emplearon los valores propuestos en la ecuación 1.

\section{Efecto del comportamiento histerético}

De acuerdo con los estudios presentados por Miranda y Ruiz (2002) y Terán (2005), referentes a realizar una estimación razonable de la resistencia lateral de diseño de estructuras ubicadas en las zona del Lago considerando las particularidades del ciclo histerético, se empleó para la obtención del espectro de diseño inelástico, un factor de corrección por comportamiento degradante (ec. 6). 
De acuerdo con Miranda y Ruiz (2002) y Terán (2005), el no considerar este efecto en estructuras que exhiben comportamiento degradante, como el caso de estructuras de concreto reforzado, puede resultar en una subestimación importante de la resistencia lateral. Por lo general, cuando el periodo del sistema $T$ es menor que el periodo del suelo $T_{g}$, el requerimiento de resistencia para un sistema de un grado de libertad (1GDL) con degradación de rigidez suele ser mayor que para un sistema con comportamiento elasto-plástico perfecto, mientras que para $T$ mayor que $T_{g}$, la resistencia para un sistema de 1GDL con degradación de rigidez es comparable o incluso menor que la de un sistema con comportamiento elasto-plástico perfecto.

El factor que considera el efecto del comportamiento degradante $\left(S_{a n}\right)$, inicialmente propuesto por Terán (Terán 2005), fue posteriormente modificado ligeramente en la propuesta del MOC-2008 para considerar que a periodo cero el valor de este factor sea unitario (figura 5, ec. 6). Asimismo, se realizó un cambió de nomenclatura identificando ahora a dicho factor como $A_{c d}$ (Tena-Colunga et al. 2009).

$$
A_{c d}=0.8+\frac{1}{2+3\left(\frac{T}{0.5 T_{s}}-1\right)^{5}}
$$

donde $T$ es el periodo del sistema en estudio y $T_{s}$ el periodo del sitio.

En la figura 6 se muestra el espectro de diseño inelástico empleado para el diseño de los edificios de 15 niveles, el cual fue reducido por conceptos de ductilidad y sobrerresistencia, y en el que se considera el efecto del factor por comportamiento degradante. Para ilustrar las diferencias en el espectro de diseño inelástico al tomar en cuenta el efecto del comportamiento degradante por medio del factor $A_{c d}$, en la figura 6 se muestra también el espectro de diseño inelástico que no considera dicho efecto. Las líneas verticales indican el intervalo de periodos en que se encuentran ubicados los modelos de 15 niveles.

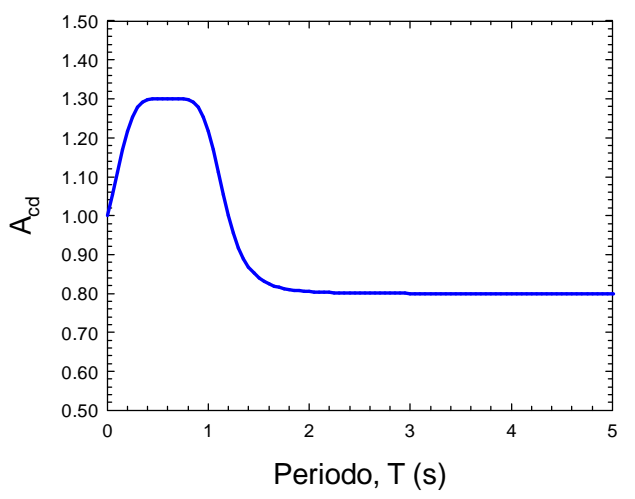

Figura 5. Variación del factor $A_{c d}$ para tomar en cuenta el efecto del comportamiento degradante al considerar $T_{s}=1.2 \mathrm{~s}$.

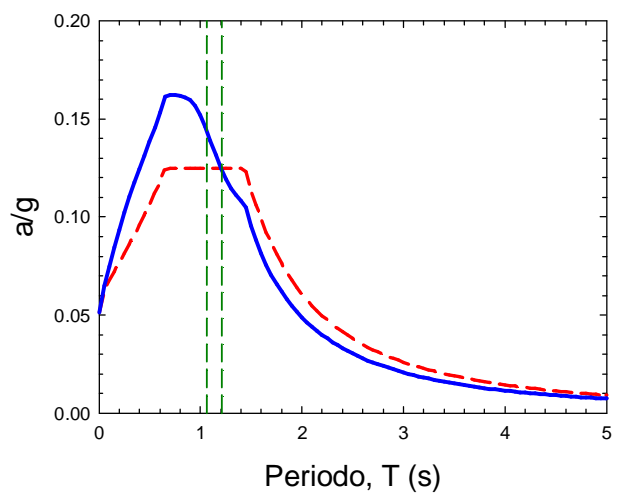

Figura 6. Espectros de diseño: 1) reducido por ductilidad y sobrerresistencia (línea discontinua) y, 2) reducido por ductilidad y sobrerresistencia y afectado por el factor de comportamiento degradante (línea continua)

\section{Edificios de 24 niveles}

Estos edificios se ubicaron para su diseño en la zona $\mathrm{III}_{\mathrm{b}}$, que representa la zona de mayores demandas sísmicas en la zona del Lago del Distrito Federal de acuerdo con la zonificación sísmica propuesta en las NTCS-04. Es importante considerar que debido a que esta zona es una de las más 
densamente instrumentadas dentro del Valle de México, existen disponibles un número importante de registros acelerométricos, los cuales fueron empleados en un proceso de escalamiento sísmico, para realizar los análisis dinámicos no lineales paso a paso. En la tabla 3 se reportan las propiedades dinámicas de los edificios de 24 niveles.

Tabla 3. Características dinámicas de los edificios 24T1 y 24T2

\begin{tabular}{|c|c|c|c|c|c|c|c|}
\hline \multirow{3}{*}{ Modo } & \multirow{3}{*}{ Dirección } & \multicolumn{3}{|c|}{ Edificio 24T1 } & \multicolumn{3}{|c|}{ Edificio $24 T 2$} \\
\hline & & \multirow{2}{*}{$\begin{array}{l}\text { Periodo } \\
\text { (s) }\end{array}$} & \multicolumn{2}{|c|}{$\begin{array}{c}\text { Masa Modal } \\
(\%)\end{array}$} & \multirow{2}{*}{$\begin{array}{l}\text { Periodo } \\
\text { (s) }\end{array}$} & \multicolumn{2}{|c|}{$\begin{array}{c}\text { Masa Modal } \\
(\%)\end{array}$} \\
\hline & & & $E-W$ & $N-S$ & & $E-W$ & $N-S$ \\
\hline 1. Primer modo traslación & E-W & 1.357 & 71.62 & 0.00 & 1.486 & 72.61 & 0.00 \\
\hline 2. Primer modo traslación & $\mathrm{N}-\mathrm{S}$ & 1.418 & 0.00 & 70.90 & 1.533 & 0.00 & 70.61 \\
\hline 3. Primer modo de rotación & & 0.952 & 0.00 & 0.00 & 1.140 & 0.00 & 0.00 \\
\hline 4. Segundo modo traslación & E-W & 0.457 & 15.25 & 0.00 & 0.523 & 14.05 & 0.00 \\
\hline 5. Segundo modo traslación & $\mathrm{N}-\mathrm{S}$ & 0.443 & 0.00 & 16.46 & 0.503 & 0.00 & 15.79 \\
\hline 6. Segundo modo de rotación & & 0.333 & 0.00 & 0.00 & 0.405 & 0.00 & 0.00 \\
\hline 7. Tercer modo traslación & E-W & 0.257 & 4.26 & 0.00 & 0.298 & 4.17 & 0.00 \\
\hline 8. Tercer modo traslación & $\mathrm{N}-\mathrm{S}$ & 0.234 & 0.00 & 4.48 & 0.277 & 0.00 & 4.54 \\
\hline 9. Tercer modo de rotación & & 0.190 & 0.00 & 0.00 & 0.235 & 0.00 & 0.00 \\
\hline$\Sigma \%$ masas modales & & & 91.12 & 91.83 & & 90.83 & 90.94 \\
\hline
\end{tabular}

Para la definición del espectro de diseño elástico, conforme a lo especificado en el Apéndice A de las NTCS-04 (AA), se consideró como periodo dominante más largo del terreno un valor $T_{s}=2.0$ segundos. Se obtuvieron además los siguientes parámetros: (a) coeficiente de aceleración del terreno $a_{0}=$ 0.25 , (b) coeficiente sísmico $c=1.20$, (c) $k=0.35 \mathrm{y}$, (d) $\operatorname{los}$ periodos característicos $T_{a}=1.175 \mathrm{~s}$ y $T_{b}=$ 2.40 s. Dado que no se consideró la interacción suelo-estructura, el factor de reducción por amortiguamiento suplementario se considero unitario $(\beta=1)$. En este caso, ya que la relación altura a base menor del edifico $\left(H / B_{\text {menor }}\right)$ excede el límite de 2.5 , establecido en las NTCS-04 para definir si la estructura es regular, se consideró un factor $\alpha=0.9$ por concepto de irregularidad por esbeltez, por lo que el espectro de diseño elástico se redujo por concepto de ductilidad empleando el factor $\alpha Q$ '.

Al igual que los edificios de 15 niveles, los edificios de 24 niveles están ubicados en la zona del lago del Distrito Federal, por lo que como se comentó, para el cálculo del espectro de diseño inelástico se consideró el factor de corrección por comportamiento degradante (ec. 6). El factor $A_{c d}$ considerado para la zona $\mathrm{III}_{\mathrm{a}}$ difiere del considerado para la zona $\mathrm{III}_{\mathrm{b}}$, pues éste está en función del periodo del sitio considerado $\left(T_{s}\right)$.

Finalmente, en la figura 7 se muestra el espectro de diseño inelástico empleado para el diseño de los edificios de 24 niveles, el cual fue reducido por conceptos de ductilidad y sobrerresistencia $\left(Q^{\prime} R\right)$, y en el cual se considera el efecto de los factores de corrección por irregularidad $(\alpha)$ y por comportamiento degradante $\left(A_{c d}\right)$. Nuevamente, para ilustrar las diferencias en el espectro de diseño inelástico al tomar en cuenta el efecto del comportamiento degradante por medio del factor $A_{c d}$, en la figura 7 se muestra también el espectro de diseño inelástico que no considera dicho efecto. Las líneas verticales indican el intervalo de periodos en que se encuentran ubicados los modelos de 24 niveles. 


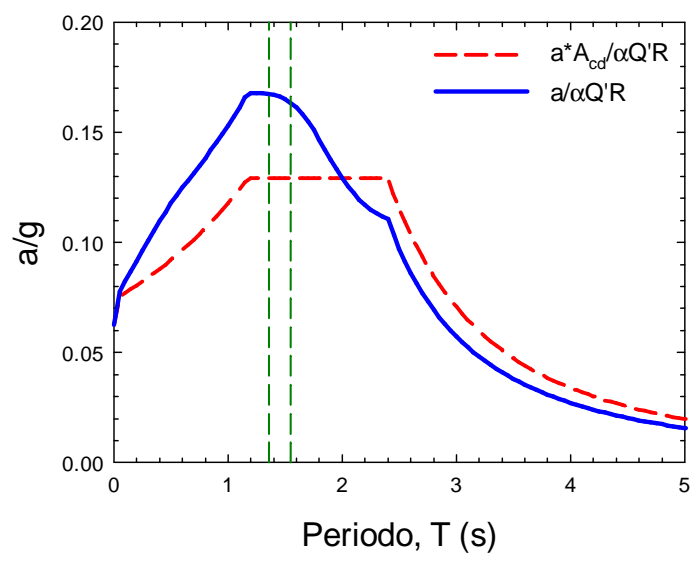

Figura 7. Espectros de diseño: 1) reducido por ductilidad y sobrerresistencia y afectado por irregularidad estructural (línea discontinua) y, 2) reducido por ductilidad y sobrerresistencia, y afectado por el factor de comportamiento degradante e irregularidad estructural (línea continua)

\section{Efectos de torsión}

Para tomar en cuenta los efectos de torsión accidental, para ambas direcciones y cada nivel, se trasladó el punto de aplicación de las cargas laterales obtenidas de los análisis modales espectrales en $\pm 0.05 \mathrm{~b}$ para el caso de los modelos ubicados en roca conforme al MOC-2008 (modelos de ocho niveles); en tanto que para los modelos de 15 y 24 niveles ubicados en terreno blando el punto de aplicación de las cargas laterales se trasladó $\pm 0.10 b$ conforme a las NTCS-04, realizando un análisis para cada cuadrante y seleccionando la condición más desfavorable (figura 8). Lo anterior representa una aproximación, mediante la aplicación de un momento estático, del acoplamiento entre los modos debido a la torsión accidental. Las combinaciones modales se realizaron de acuerdo a las reglas de combinación modal SRSS o CQC, según correspondiera a cada caso.

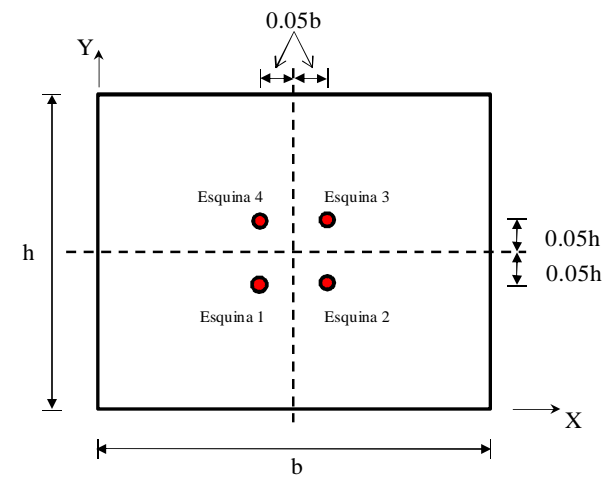

a) Modelos de ocho niveles (MOC-2008)

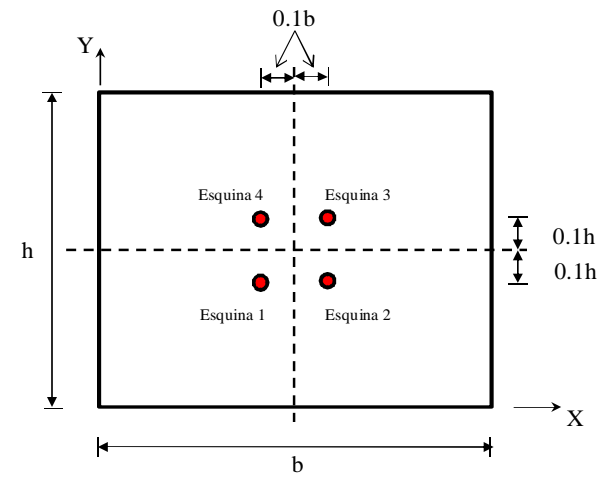

b) Modelos de 15 y 24 niveles (NTCS-04)

Figura 8. Ubicación de los puntos de aplicación de las cargas laterales para tomar en cuenta los efectos de torsión accidental de acuerdo con las NTCS-04 


\section{CONSIDERACIONES PARA LOS ANÁLISIS NO LINEALES}

Para efectuar los análisis dinámicos no lineales paso a paso se empleó el programa RUAUMOKO (Carr 2004), utilizando modelos con distribución de fuerzas laterales entre los marcos por acción del diafragma rígido. Las vigas y columnas se modelaron mediante elementos tipo viga-columna de su librería (Giberson BEAM member y concrete BEAM-COLUMN member, respectivamente), que consideran la posibilidad de formar rótulas plásticas en sus extremos, definiendo en cada caso las superficies de falla conforme lo recomienda dicho programa. El comportamiento histerético tanto de vigas como de columnas se definió por medio del modelo modificado de Takeda con base en lo propuesto por Otani en 1974 (modelo de histéresis número cuatro en la librería de Ruaumoko). Con lo anterior, es posible considerar la degradación de rigidez en los componentes de concreto reforzado, aspecto de suma importancia en la interpretación de los análisis dinámicos a realizar, por ejemplo, en la estimación de la degradación de rigidez en cada entrepiso. Los parámetros que definen la degradación de rigidez $(\alpha \mathrm{y} \beta)$ en el modelo modificado de Takeda difieren para el caso de vigas respecto al de columnas, pues en el caso de estas últimas, la degradación de rigidez que se espera es menor, pues el núcleo de concreto tiene un confinamiento mayor al considerado en las vigas (tabla 4). La influencia de los factores $\alpha$ y $\beta$ en el modelo de Takeda, así como los valores considerados en los análisis se comentan con detalle en Godínez (2010).

Asimismo, las diagonales metálicas de contraventeo se modelaron con el elemento tipo vigacolumna de su librería (Steel BEAM-COLUMN member) considerando ambos extremos del elemento articulados. En este caso, el comportamiento histerético se definió empleando el modelo propuesto por Remennikov (Remennikov y Walpole 1997), modelo de histéresis número 24 en la librería de Ruaumoko. El modelo propuesto por Remennikov y Walpole (1997) tiene la capacidad de representar de manera adecuada el comportamiento cíclico de elementos sujetos a cargas axiales. Los autores de referencia sustentan su investigación con base en la comparación de los resultados analíticos obtenidos con resultados de estudios analíticos-experimentales derivados de estudios previamente desarrollados por Black et al. (1980) e Ikeda y Mahin (1984). De hecho, el modelo propuesto por Remennikov y Walpole (1997) se basa en gran medida en los resultados de los estudios comentados. En la tabla 5 se muestran los parámetros considerados para la definición del módulo tangente $\left(\mathrm{e}_{1}, \mathrm{e}_{2}, \mathrm{e}_{3}, \mathrm{e}_{4}\right)$ en la definición del modelo de Remennikov, los cuales corresponden a relaciones de esbeltez del contraviento $70 \leq k L / r \leq 90$. La descripción de los parámetros y características empleados para la definición del modelo de Remennikov se comentan con detalle en Godínez (2010).

Tabla 4. Parámetros $\alpha$ y $\beta$ empleados en vigas y columnas para la definición del modelo de Takeda

\begin{tabular}{ccc}
\hline Elemento tipo & $\boldsymbol{\alpha}$ & $\mathbf{B}$ \\
\hline Vigas & 0.10 & 0.45 \\
Columnas & 0.10 & 0.60 \\
\hline
\end{tabular}

Tabla 5. Valores empleados en los análisis para la definición del módulo tangente en contravientos

\begin{tabular}{cc}
\hline Parámetro & Valor considerado en el análisis \\
\hline$e_{1}$ & 0.15 \\
$e_{2}$ & 0.90 \\
$e_{3}$ & 1.20 \\
$e_{4}$ & 0 \\
\hline
\end{tabular}


En los análisis se consideró un amortiguamiento viscoso equivalente del $5 \%$, en congruencia con lo supuesto en la definición de los espectros de diseño sísmico de las NTCS-04 y el MOC-2008. Se consideraron los efectos P- $\Delta$ y se ignoró la interacción suelo-estructura.

En todos los análisis no lineales realizados se consideró que en los elementos existen fuentes de sobrerresistencia, derivadas de efectos tales como: la losa y su acero de refuerzo, considerar esfuerzos de fluencia reales en el acero de refuerzo, entre otros. Los criterios para la determinación de resistencias al incluir fuentes adicionales de sobrerresistencia se comentan con detalle en Godínez y Tena (2007) y Godínez (2010).

Con la finalidad de contar con una base de datos suficiente para el cálculo de valores promedio, para cada zona de estudio se consideraron como mínimo nueve registros acelerométricos. Los acelerogramas empleados en los análisis dinámicos no lineales son representativos y de características similares al sismo máximo contemplado por los Reglamentos, con base en los cuales se realizaron cada uno de los diseños, como se discute posteriormente.

En las figuras 9 y 10 se muestran, para cada edificio, los modelos empleados en los análisis no lineales paso a paso, en los que se considera existe distribución de fuerzas laterales entre los marcos por acción del diafragma rígido. Se muestran únicamente los modelos de ocho niveles, ya que los modelos de 15 y 24 niveles cuentan con la misma configuración.

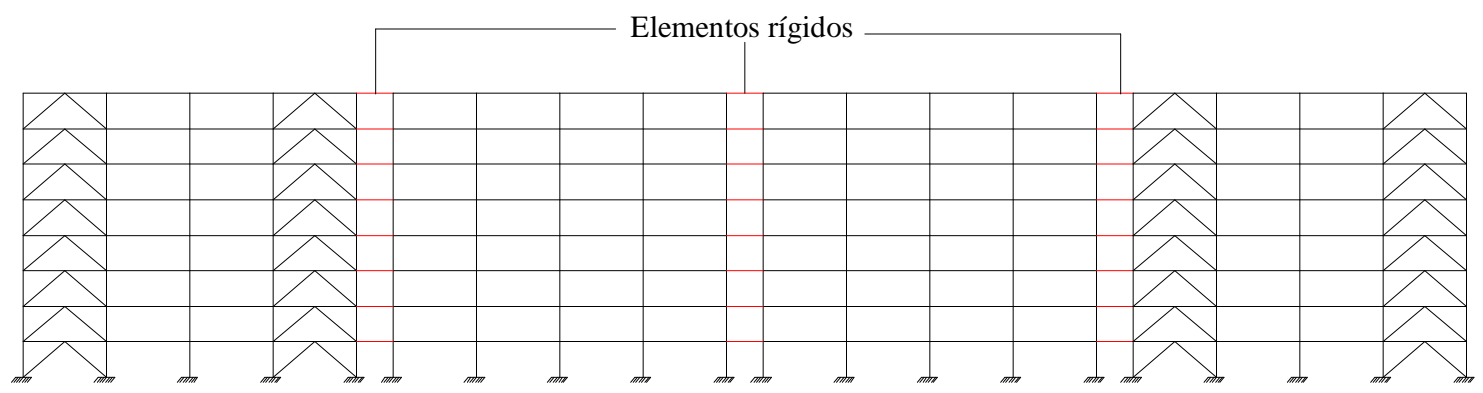

a) Modelo en dirección $X$

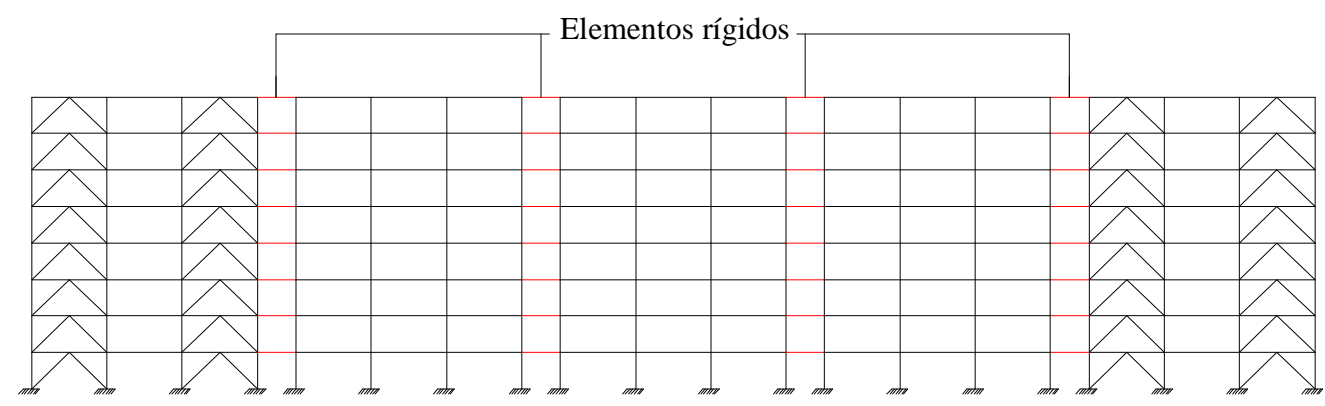

b) Modelo en dirección $Y$

Figura 9. Modelos con distribución de fuerzas laterales entre marcos por acción del diafragma rígido de los edificios tipo 1 


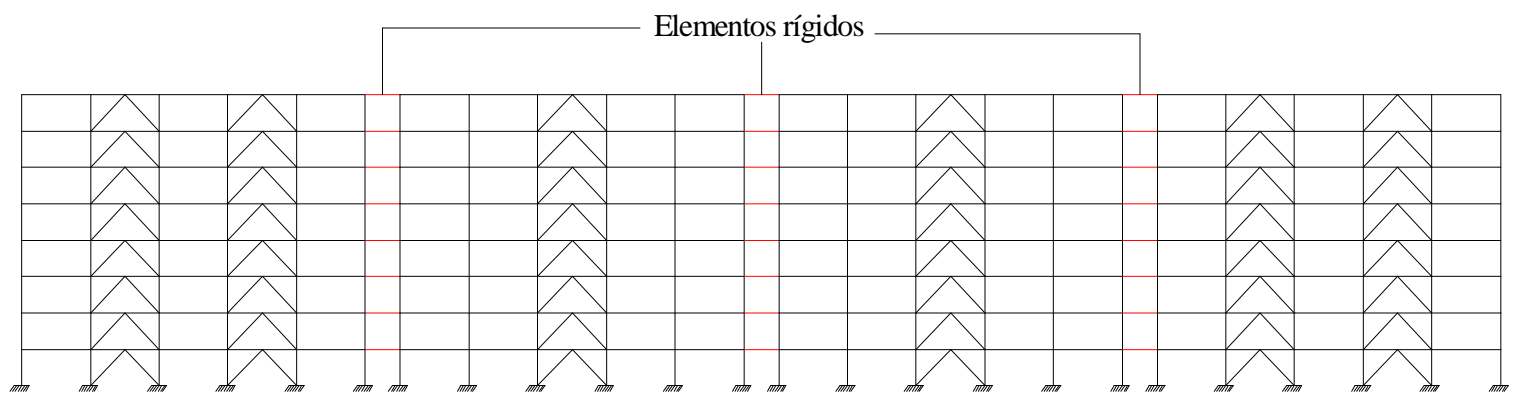

a) Modelo en dirección $X$

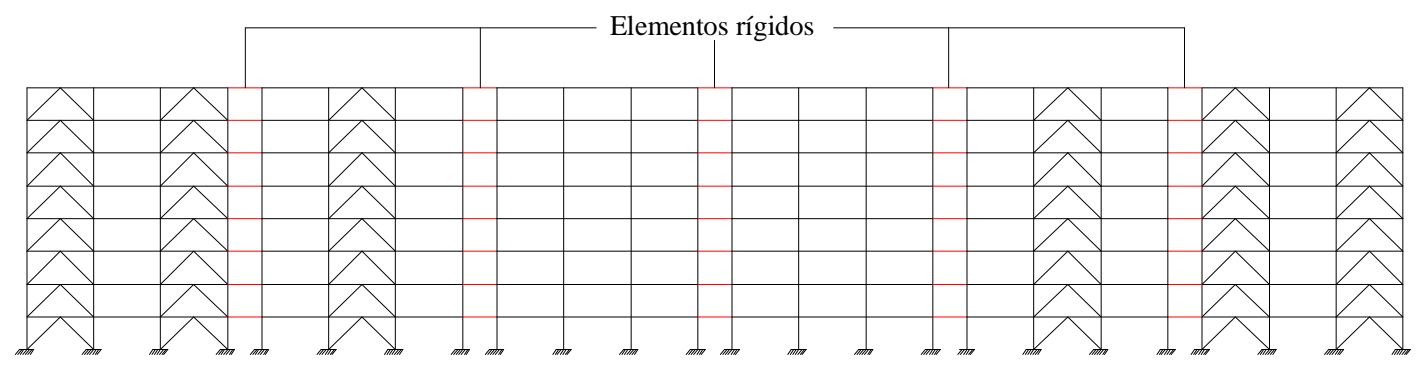

b) Modelo en dirección $Y$

Figura 10. Modelos con distribución de fuerzas laterales entre marcos por acción del diafragma rígido de los edificios tipo 2

\section{Movimientos del terreno}

Para cada una de las zonas en que se ubicaron los edificios diseñados: (a) costa de Guerrero (edificios de ocho niveles), (b) zona $\mathrm{III}_{\mathrm{a}}$ del Lago del Distrito Federal (edificios de 15 niveles) y, (c) zona III $_{\mathrm{b}}$ del Lago del Distrito Federal (edificios de 24 niveles), se generaron acelerogramas sintéticos que representan el peligro sísmico actualmente especificado en los códigos empleados para el diseño de los edificios, en este caso el Manual de Obras Civiles de la Comisión Federal de Electricidad (MOC-2008) y el Reglamento de Construcciones del Distrito Federal (RCDF-04). Lo anterior debido a que los registros acelerométricos de los eventos sísmicos con que se cuenta no representan al 100\% el peligro sísmico actualmente considerado en el MOC-2008 o las NTCS-04. El proceso de escalamiento empleado toma como base la información disponible de registros acelerométricos de eventos sísmicos pasados.

El procedimiento planteado para la obtención de los acelerogramas sintéticos, contempla el empleo de las funciones de transferencia empíricas promedio (FTE) obtenidas por Pérez-Rocha (1998) a partir de los registros de la red acelerométrica de la ciudad de México (figura 11), y el espectro promedio de amplitudes de Fourier (EAF) correspondiente a terreno firme. En este caso particular, se tomó el promedio de terreno firme como el promedio observado en CU. La metodología de escalamiento sísmico empleada, se discute con detalle en Pérez-Rocha (1998), Godínez (2005), Godínez et al. (2005) y Tena-Colunga et al. (2007).

Para conformar la base de acelerogramas sintéticos, primeramente se identificaron las estaciones acelerométricas ubicadas en las zonas de interés. Con ese fin, en la figura 11 se muestran las estaciones 
acelerométricas de las cuales se tiene información, así como su ubicación de acuerdo con la zonificación sísmica de las NTCS-04. Se muestra también, mediante contornos con línea azul y verde, las zonas con daños y mayores daños registradas durante los sismos de septiembre de 1985, las cuales cubren parte importante de las zonas III $_{\mathrm{a}}$ y III $_{\mathrm{b}}$.

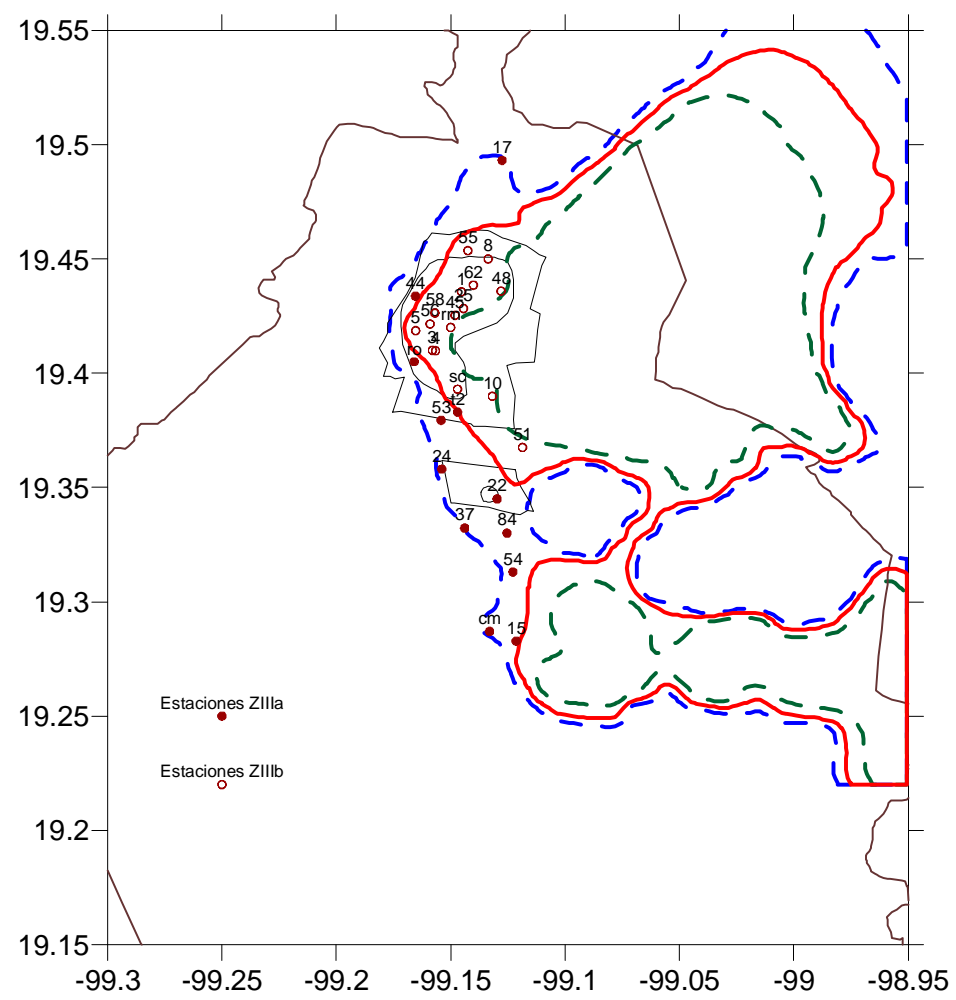

Figura 11. Ubicación geográfica de estaciones acelerométricas de la RACM conforme la zonificación sísmica de las NTCS-04

Una vez identificadas y seleccionadas las estaciones acelerométricas a considerar, se realizaron para cada estación, simulaciones numéricas de tal manera que se obtuvieran registros acelerométricos congruentes con el peligro sísmico de la zona estudiada, es decir, que la aceleración espectral máxima obtenida en cada caso sea equiparable con la estipulada en el espectro de diseño elástico de la zona considerada.

En la figura 12 se compara el espectro de respuesta elástico de cada acelerograma sintético respecto al espectro de diseño elástico de cada zona considerada, de acuerdo con las NTCS-04 y el MOC-2008. Se observa que los espectros de respuesta asociados a los acelerogramas generados de forma artificial son congruentes con el peligro sísmico considerado en ambos reglamentos. Asimismo, en la figura 13 se muestran algunos de los acelerogramas sintéticos correspondientes a la zona costa de Guerrero y la zona $\mathrm{III}_{\mathrm{a}}$, los cuales fueron empleados para realizar los análisis dinámicos no lineales paso a paso de los modelos de los edificios de 8 y 15 niveles, respectivamente.

Los registros de aceleración obtenidos mediante el procedimiento mencionado fueron filtrados y corregidos por línea base previo a su empleo en los análisis dinámicos no lineales. Con esto se evitan errores numéricos que llevan a que los desplazamientos asociados sean irreales, lo cual no es apreciable a simple vista. 


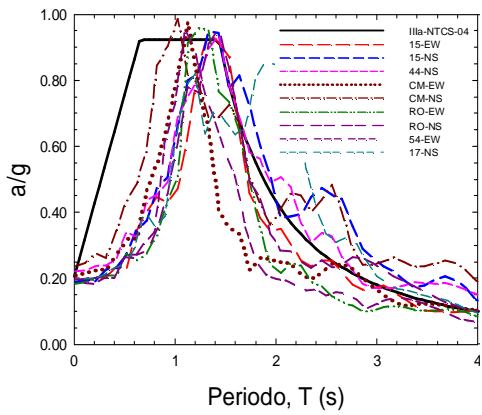

a) Zona $\mathrm{III}_{\mathrm{a}}$

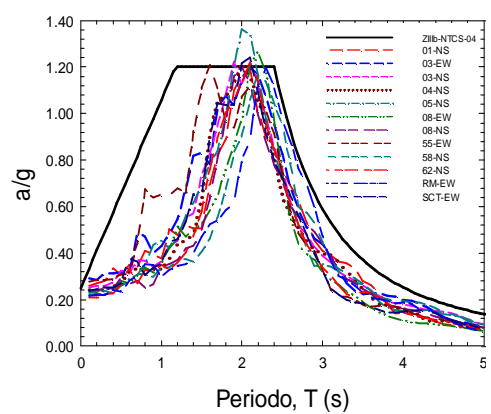

b) Zona $\mathrm{III}_{\mathrm{b}}$

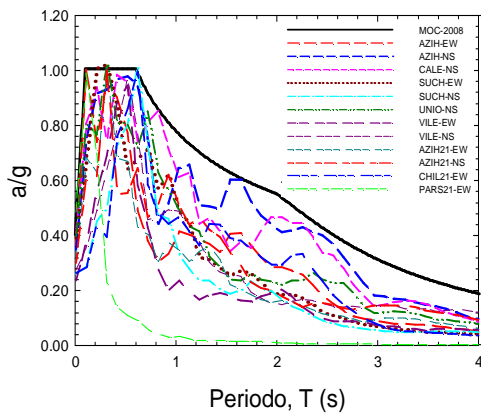

c) Zona costa de Guerrero

Figura 12. Comparativa entre los espectros de respuesta elásticos de los acelerogramas sintéticos y el espectro de diseño elástico de cada zona considerada

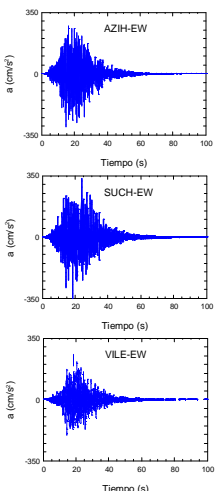

a) Zona costa de Guerrero
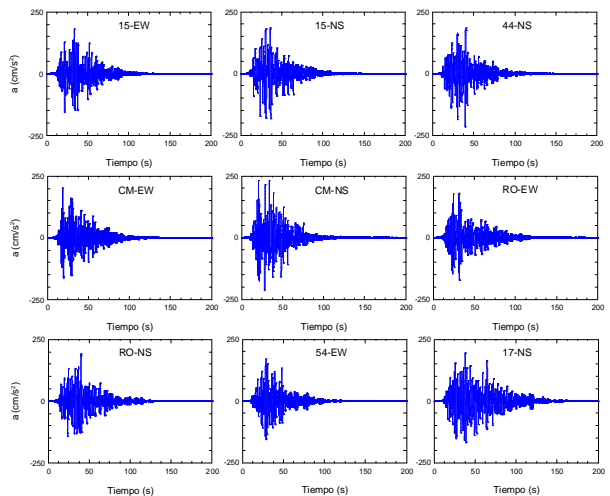
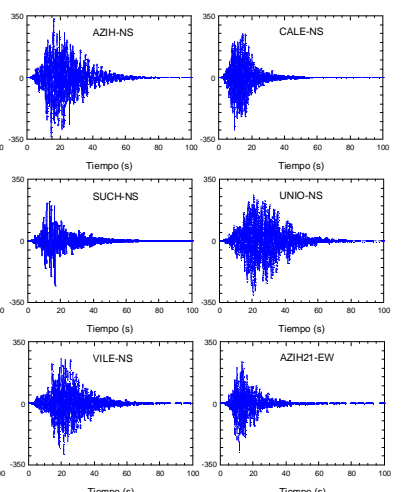

\section{(1)}

Figura 13. Acelerogramas sintéticos considerados en los análisis dinámicos no lineales paso a paso de los modelos de a) ocho niveles y b) 15 niveles

\section{RESULTADOS DE LOS ANÁLISIS DINÁMICOS NO LINEALES}

Para todos los modelos estudiados mediante análisis dinámicos no lineales paso a paso ante cada uno de los acelerogramas considerados, se estudiaron los siguientes parámetros: (a) curvas de histéresis de entrepiso y globales, (b) mapeo de rotaciones plásticas normalizadas, (c) distorsiones de entrepiso asociada a la primera fluencia de cualquier elemento estructural que forma parte del entrepiso $\left(\Delta_{\mathrm{fe}}\right)$, (d) distorsiones de fluencia $\left(\Delta_{\mathrm{y}}\right)$, calculadas con base en un ajuste elasto-plástico, (e) envolventes de distorsiones de entrepiso máximas $\left(\Delta_{\max }\right)$, (f) número de ciclos en que se presenta respuesta inelástica, $(\mathrm{g})$ cociente del promedio de las rigideces de entrepiso secantes asociadas a los medios ciclos histeréticos inelásticos $\left(\mathrm{K}_{\text {prom }}\right)$ y la rigidez elástica de entrepiso $\left(\mathrm{K}_{\mathrm{el}}\right)$, (h) demandas máximas de ductilidad de entrepiso y globales $(\mu)$, (i) cortantes de entrepiso máximos de columnas y diagonales $\left(\mathrm{V} / \mathrm{W}_{\mathrm{T}}\right)$, (j) sobrerresistencia máxima demandada por los movimientos del terreno considerados. En la figura 14 se ilustran esquemáticamente algunos de estos conceptos.

En esta sección, por cuestiones de espacio, se muestran mayoritariamente los resultados de los modelos en que se observó la mayor demanda inelástica para cada altura considerada. Los resultados 
detallados de cada modelo ante cada acelerograma se reportan en Godínez (2010). Se muestran en primera instancia las curvas de histéresis y los mapeos de rotaciones plásticas normalizadas asociadas al acelerograma que indujo las mayores demandas en cada modelo. Posteriormente, se resumen las respuestas máximas y globales, cuyos parámetros se definen en la figura 14.
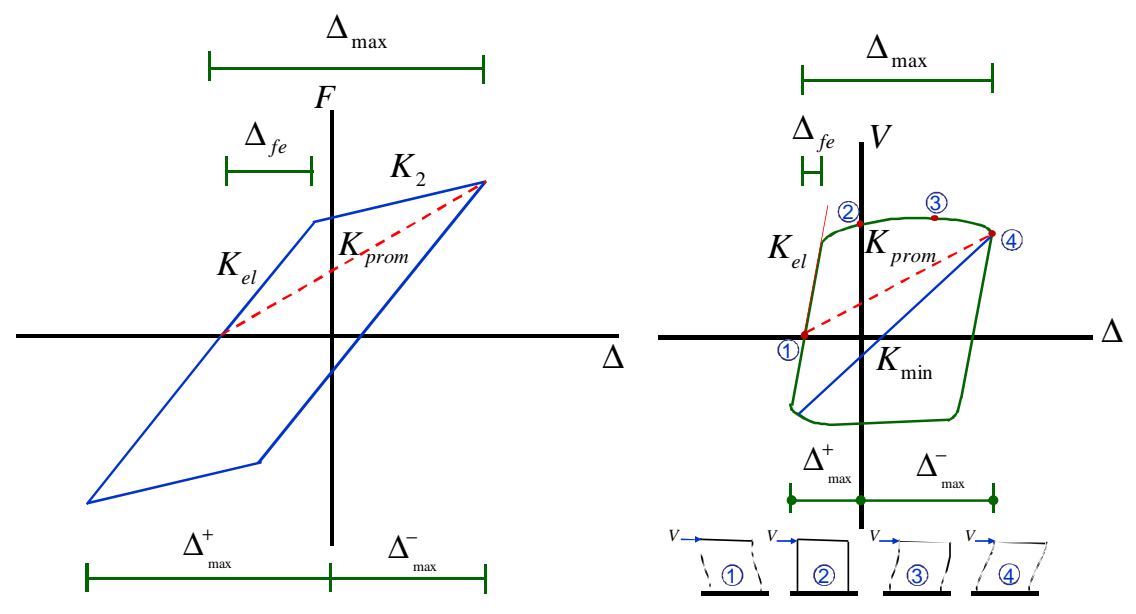

Figura 14. Definición de parámetros a partir de ciclos y medios ciclos de histéresis, generalmente asimétricos (adaptada de Tena y Correa 2008)

\section{Modelos de ocho niveles}

De las curvas de histéresis de los modelos correspondientes al edificio tipo dos (modelos 8XT2 y 8YT2, figura 15) se observa que, a pesar de considerar registros acelerométricos asociados con el espectro de diseño con base en el cual se realizaron los diseños de los edificios de esta altura (espectro en roca de acuerdo al MOC-2008), no existió una demanda de comportamiento inelástico importante. El mismo efecto se observó en los modelos del edificio tipo 1.

De los resultados correspondientes a los modelos del edificio tipo 2, se observó un mayor trabajo inelástico en el modelo en dirección $X$ (modelo 8XT2, figura 15a), así como demandas de distorsión en los entrepisos tres a cinco ligeramente superiores a las observadas en la dirección $Y$ (modelo 8YT2, figura 15b). Congruente con esto, la degradación de rigidez en dirección $X$ fue más notoria que en dirección $Y$. Como se observa tanto de las curvas de histéresis como de los mapeos de rotaciones plásticas normalizadas (figuras 15 y 16), los dos últimos entrepisos permanecen elásticos. Este efecto se observó también en los modelos del edificio tipo 1. Por cuestiones de espacio, en esta sección se presentan mayoritariamente los resultados del modelo 8XT2, en el que se observó una mayor demanda de comportamiento inelástico.

Como se observa de las figuras 15a y 16, el mayor trabajo inelástico en el modelo 8XT2 se presentó en los entrepisos dos a cinco. Se observa que la mayoría de los contravientos ubicados en estos entrepisos trabajan inelásticamente; sin embargo, nuevamente debido a las bajas demandas de distorsión impuestas por los movimientos del terreno, no existe pandeo. En el modelo 8XT2, a diferencia del modelo 8XT1, se observó un trabajo inelástico de las vigas de los entrepisos tres a cinco más uniforme en todos los marcos, efecto debido probablemente a la mejor distribución de rigideces, la cual en el caso del modelo 8XT2 es más uniforme al estar todos los marcos contraventeados. En todos los modelos de ocho niveles, no se observaron fluencias en columnas en ninguna dirección, cumpliendo con ello el mecanismo de colapso último supuesto en el diseño (figura 16). 

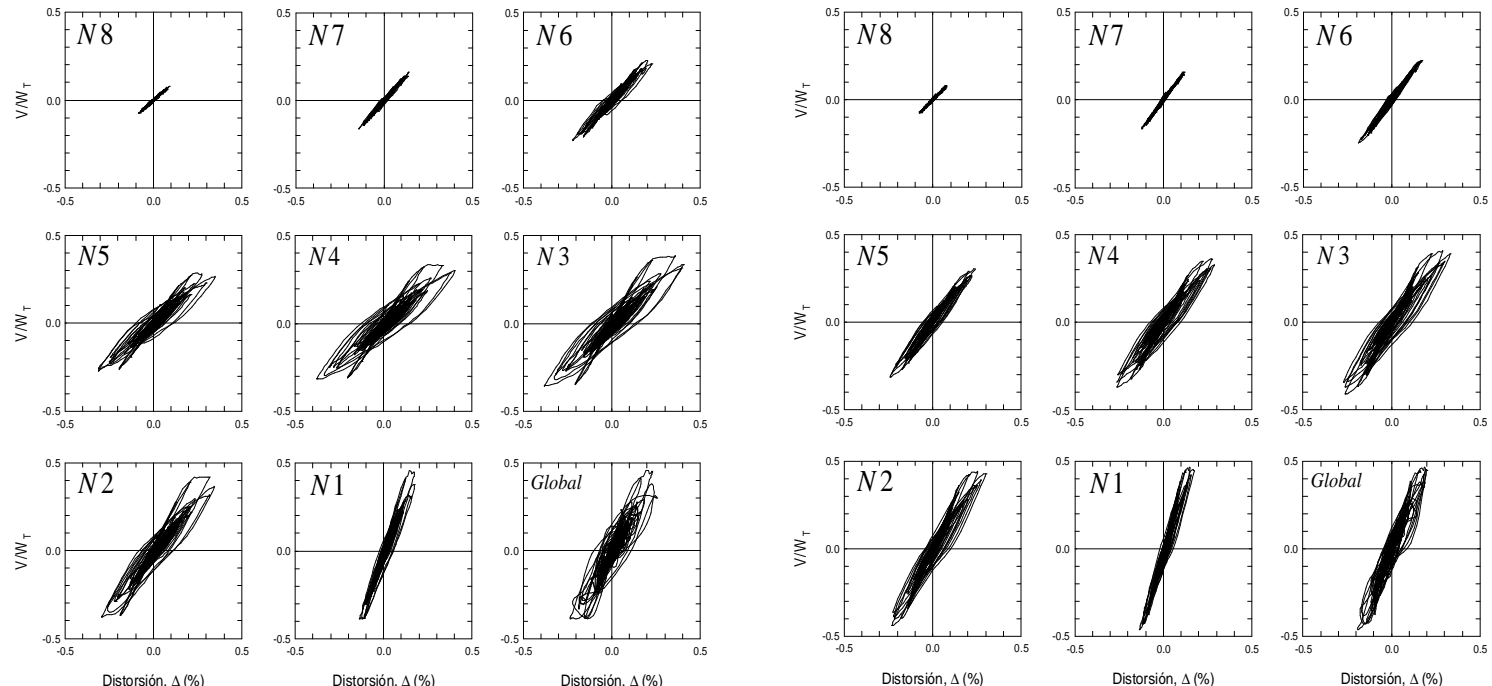

a) Modelo 8XT2
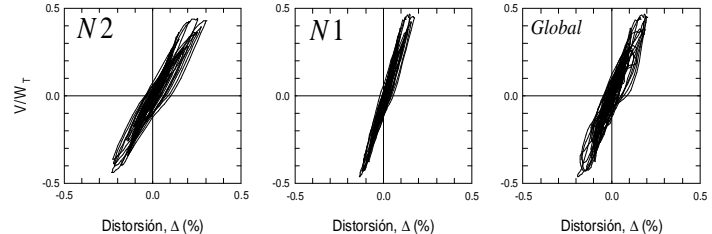

b) Modelo 8YT2

Figura 15. Curvas de histéresis de los modelos de ocho niveles correspondientes al edificio tipo 2 ante el acelerograma AZIH-NS que generan las demandas máximas, considerando sobrerresistencia.

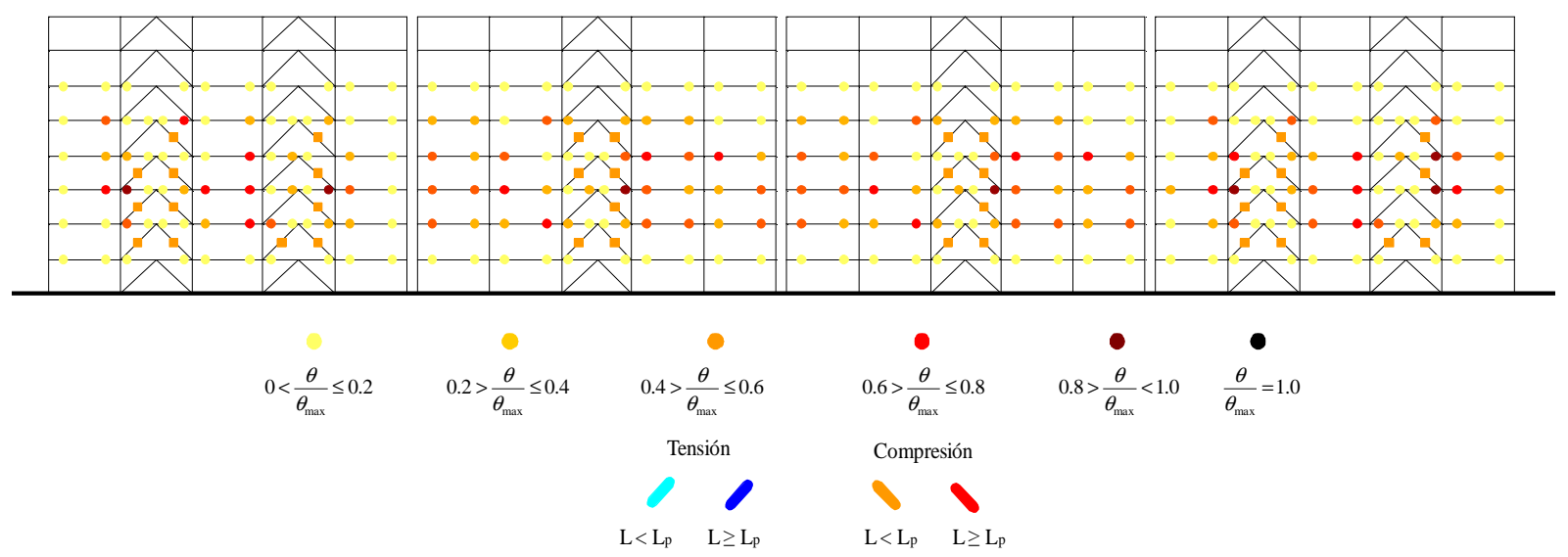

Figura 16. Rotaciones plásticas normalizadas del modelo 8XT2 ante el acelerograma AZIH-NS que genera las demandas máximas, considerando sobrerresistencia.

Asimismo, de las gráficas que resumen las respuesta máxima (símbolos llenos) y promedio (símbolos vacíos) de los modelos 8XT2 y 8YT2 (figuras $17 \mathrm{~b}$ y 18 b), se observa que las distorsiones de fluencia máximas, calculadas a partir de un ajuste elasto-plástico de las curvas de histéresis de entrepiso y globales, concuerdan de manera razonable con el límite propuesto para la revisión del estado límite de servicio $\left(\Delta_{y}=0.002\right)$, lo cual también ocurre en el resto de los modelos de ocho niveles. Lo anterior indica que para edificios de esta altura, el límite propuesto para la revisión del estado límite de servicio, con base en los resultados de los análisis estáticos no lineales, es adecuado. De hecho, el promedio de los entrepisos que tienen comportamiento marcadamente inelástico, exceptuando el primero por la condición impuesta de frontera de base rígida, es $\Delta_{y p r o m}=0.002,0.0019,0.0018$ y 0.0017 para los modelos 8XT1, 8YT1, 8XT2 y 8 YT2, respectivamente. 
De las figuras $17 \mathrm{c}$ y $18 \mathrm{c}$, se observa tanto de la respuesta máxima (símbolos llenos) como de la promedio (símbolos vacios), que el mayor número de ciclos inelásticos en ambos modelos se presentó en los entrepisos dos a cinco (se observó una distribución y número de ciclos inelásticos muy similar en los modelos del edificio tipo 1), presentándose para todos los modelos el mismo efecto en relación a que los ciclos inelásticos corresponden a ciclos de amplitud pequeña. Como se comentó, se observó una respuesta inelástica mayor en la dirección $X$ respecto a la dirección $Y$, lo cual es más notorio al considerar las respuestas máximas, en las que se observan demandas de ductilidad de entrepiso pico ligeramente mayores a dos (figura 17f), contrario a lo observado en el modelo en dirección $Y$ (figura 18f), en que las demandas máximas de ductilidad fueron inferiores a dos en todos los entrepisos.
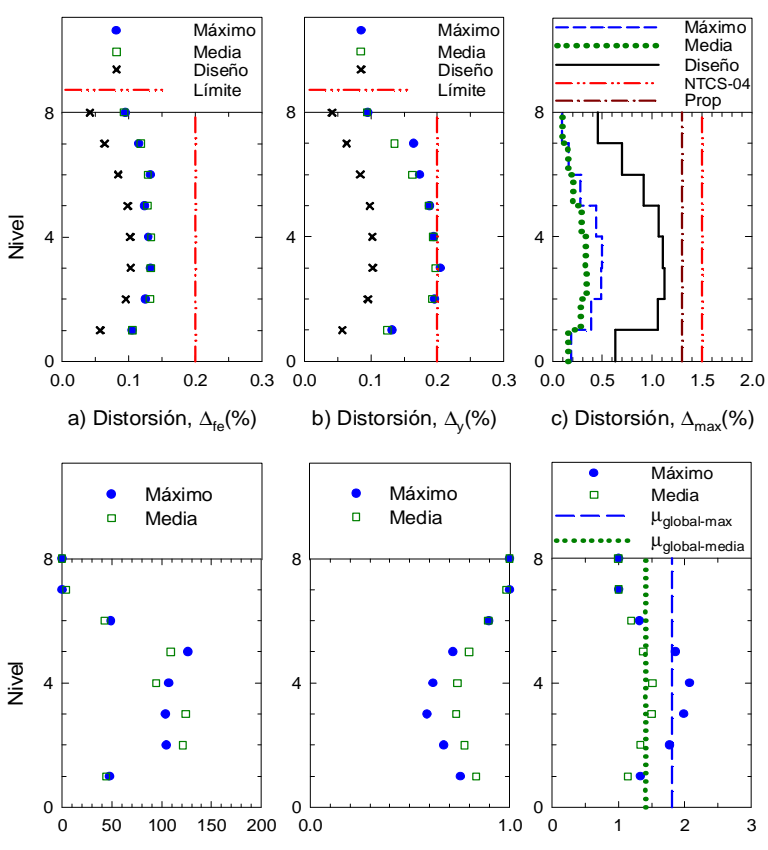

$$
\text { d) Ciclos inelásticos }
$$

e) $\mathrm{K}_{\text {prom }} / \mathrm{K}_{\mathrm{e}}$

f) Dem. de ductilidad, $\mu$
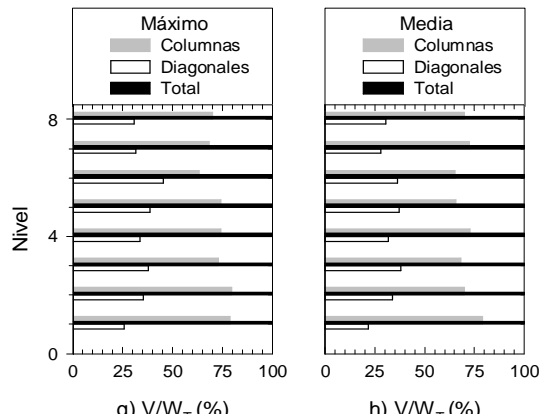

Figura 17. Respuestas máxima y promedio del modelo 8XT2 considerando sobrerresistencia
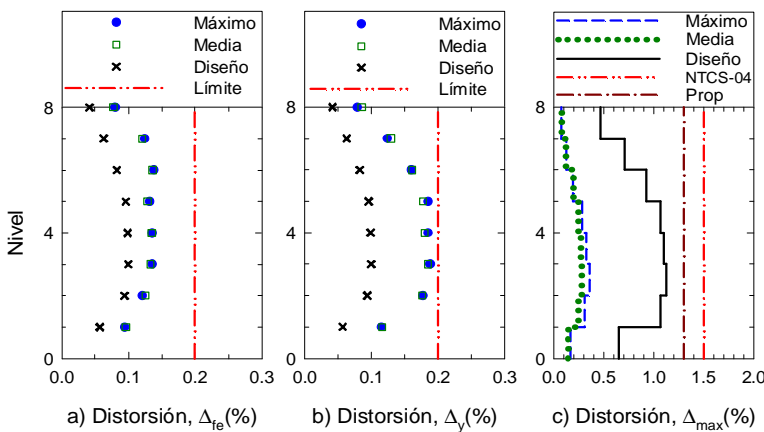

b) Distorsión, $\Delta_{y}(\%)$

c) Distorsión, $\Delta_{\max }(\%)$
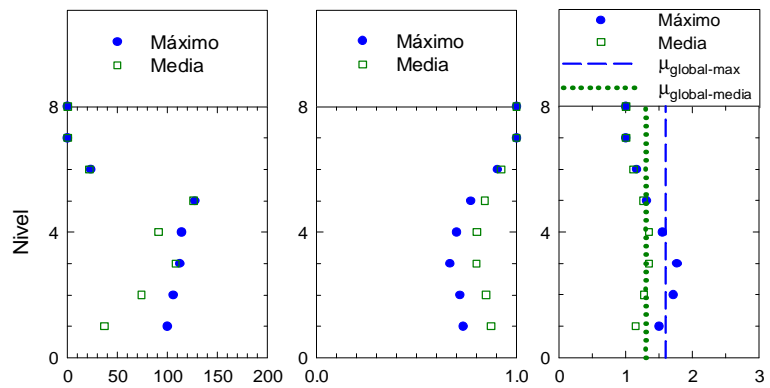

d) Ciclos inelásticos

e) $\mathrm{K}_{\text {prom }} / \mathrm{K}_{\mathrm{el}}$

f) Dem. de ductilidad, $\mu$

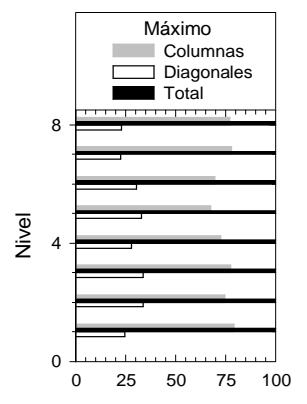

g) $V / W_{T}(\%)$

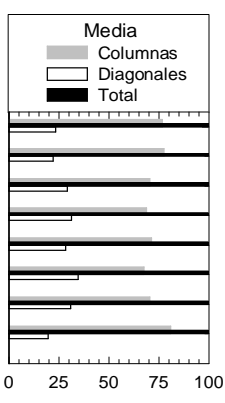

h) $\mathrm{V} / \mathrm{W}_{\mathrm{T}}(\%)$
Figura 18. Respuestas máxima y promedio del modelo 8YT2 considerando sobrerresistencia

Congruente con lo previamente comentado, de las respuestas promedio (símbolos vacíos), se observaron mayores niveles de degradación de rigidez en dirección $X$, obteniendo valores mínimos del factor $K_{\text {prom }} / K_{e l}$ cercanos a 0.7 (figura 17e), en tanto que en dirección $Y$ se obtuvieron valores mínimos del factor $K_{p r o m} / K_{e l}$ cercanos a 0.8 (figura $18 \mathrm{e}$ ). 
Resulta evidente que las demandas de ductilidad globales, obtenidas a partir de las envolventes de respuestas promedio y máxima, son inferiores a la capacidad de deformación considerada en el diseño $(Q=4)$, efecto presente en todos los modelos de ocho niveles. En dirección $X$ (figura 17f), la demanda de ductilidad global promedio $\left(\mu_{\text {global-media }}=1.40\right)$ resultó superior a la obtenida en dirección $Y\left(\mu_{\text {global }}\right.$. media $=1.32$, figura 18f), observándose el mismo efecto al considerar las respuestas máximas. Como es normal, tanto los niveles de degradación de rigidez, como las demandas de ductilidad son mayores al considerar las envolventes de respuesta máxima respecto a las obtenidas con base en las respuestas promedio.

En todos los modelos de esta altura, el análisis conjunto de estos tres parámetros (número de ciclos inelásticos, degradación de rigidez, y demandas de ductilidad), indica que los ciclos de comportamiento inelástico, a pesar de ser numerosos, son de amplitud pequeña, pues los niveles de degradación de rigidez y las demandas de ductilidad no son elevados. En la interpretación de los resultados debe considerarse que, en este caso, la degradación de rigidez obtenida es superior a la que se obtendría si se considerara un comportamiento elasto-plástico perfecto, tal y como se ha considerado en otras investigaciones para el estudio del comportamiento de edificios de concreto reforzado con base en marcos momento-resistentes (Correa 2005, Gatica 2007, Tena-Colunga et al. 2008). Como se mencionó, en este estudio para el modelado del comportamiento cíclico de los elementos de concreto reforzado, se empleó el modelo de Takeda que considera la degradación de rigidez que un elemento sufre ante cargas cíclicas. Asimismo, el modelo de Remennikov, empleado para el modelado de los contravientos de acero, considera la degradación de rigidez y resistencia posterior al pandeo.

Respecto a los cortantes de entrepiso máximos en columnas y contravientos $\left(\mathrm{V} / \mathrm{W}_{\mathrm{T}}\right.$, figuras $17 \mathrm{~g}$ y 18g), como era de esperarse, en los modelos 8XT2 y 8YT2 se presentaron aportes del sistema de contraventeo mayores que en los modelos tipo 1, pues cuentan con un número mayor de marcos contraventeados. En ambos casos, se obtuvieron valores congruentes con los inicialmente planteados en la etapa de diseño, en que se estimó que las columnas aportarían aproximadamente el $70 \%$ de la resistencia a cortante de entrepiso.

Las incertidumbres existentes en la determinación del aporte final que a la resistencia a fuerza cortante brinda el sistema de contraventeo son de esperarse, pues como se reporta en estudios previos (Godínez y Tena 2009, Godínez 2010), de los resultados de los análisis estáticos no lineales se observó una diferencia entre el aporte final predicho y el calculado. Sin embargo, como se observó de los modelos en estudio, la variación entre los valores predichos y los calculados es pequeña en la mayoría de los casos.

\section{Modelos de 15 niveles}

A diferencia de los modelos de ocho niveles, en todos los modelos de 15 niveles se observó un mayor trabajo inelástico. De la figura 19, en que se muestran las curvas de histéresis de los modelos correspondientes al edificio tipo 2, se observó que en la dirección $X$ (modelo 15XT2) existió un mayor trabajo inelástico, así como demandas de distorsión en los entrepisos cuatro a ocho ligeramente mayores a las observadas en la dirección $Y$. Como se observa tanto de las curvas de histéresis (figura 19), como de los mapeos de rotaciones plásticas normalizadas (figura 20), el comportamiento inelástico en dirección $X$ se distribuye más uniformemente en elevación, pues únicamente los dos últimos entrepisos permanecen elásticos, en tanto para la dirección $Y$, los últimos tres entrepisos permanecieron básicamente elásticos. Dado que las mayores demandas de comportamiento inelástico se observaron en el modelo 15XT2, se presentan mayoritariamente los resultados de este modelo. 
El mayor comportamiento inelástico del modelo 15XT2 se presenta en los entrepisos tres a nueve. Se observa que la mayoría de los contravientos trabajan inelásticamente, presentándose el pandeo de la mayoría de estos elementos (figura 19a). Congruente con las curvas de histéresis, en los mapeos de rotaciones plásticas (figura 20a) se observa que las vigas de los entrepisos cuatro a ocho son las que mayor demanda de trabajó inelástico presentan.
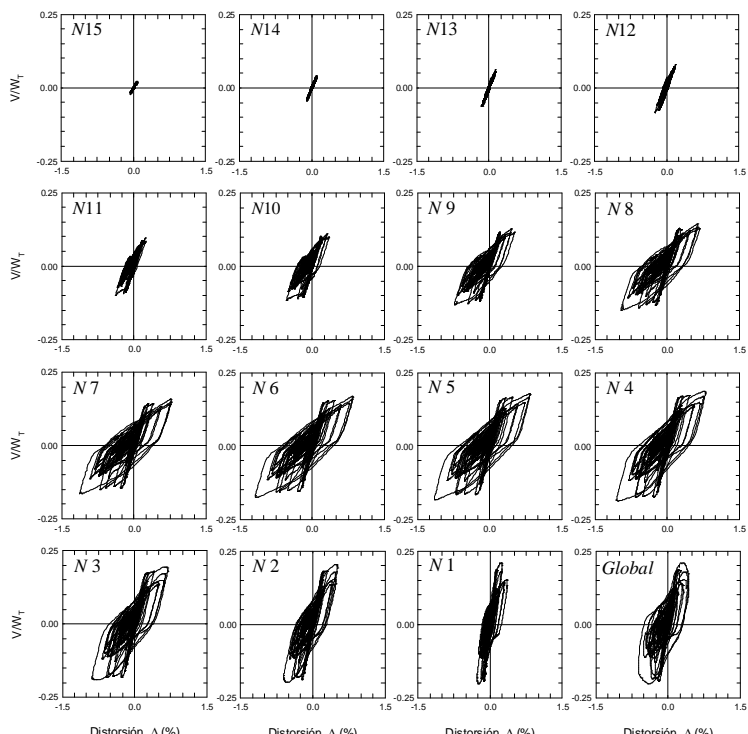

a) Modelo 15XT2 ante el acelerograma 15-EW
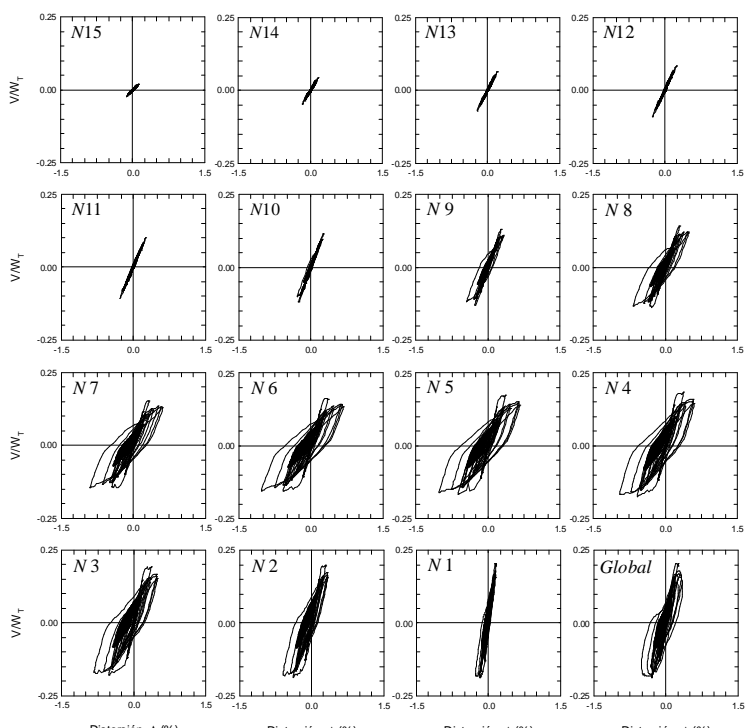

b) Modelo 15YT2 ante el acelerograma RO-NS

Figura 19. Curvas de histéresis de los modelos correspondientes al edificio tipo 2 ante los acelerogramas que generan las demandas máximas, considerando sobrerresistencia.

De forma similar, el mayor comportamiento inelástico en el modelo 15YT2 se presenta en los entrepisos tres a ocho (figura 19b). Se observa que los contravientos de este modelo ubicados en los entrepisos dos a 11 trabajan inelásticamente, presentándose el pandeo de la mayoría de estos elementos, principalmente los ubicados en los entrepisos dos a nueve (figura 20b). Congruente con las curvas de histéresis, en los mapeos de rotaciones plásticas se observa que las vigas de los entrepisos tres a ocho son las que mayor demanda de trabajó inelástico presentan.

De los mapeos de fluencias de los modelos 15XT2 y 15YT2 (figuras 20a y 20b), se observaron fluencias en la base de las columnas de planta baja, lo cual es aceptable dadas las demandas de distorsión impuestas por los movimientos del terreno y a la condición de empotramiento perfecto considerada en los análisis. Asimismo, se observan algunas fluencias en las columnas de borde de los marcos contraventeados, con rotaciones de magnitud significativamente menor a las obtenidas en vigas. Estas fluencias en columnas (columnas del tercer entrepiso) están directamente relacionadas con el cambio en el refuerzo longitudinal en las columnas, que se realiza en dicho entrepiso. Nuevamente, lo anterior podría indicar que, aunque existan demandas de refuerzo inferiores a los obtenidos en los dos primeros entrepisos, el refuerzo longitudinal de las columnas debería permanecer uniforme en una longitud mayor a la considerada en los diseños. Un comportamiento semejante se observó en los modelos del edificio tipo uno.

Finalmente, al igual que para los modelos de ocho niveles, se puede concluir para todos los modelos de 15 niveles, que los mapeos de rotaciones y deformaciones plásticas muestran que se satisface muy 
razonablemente la premisa de diseño de mecanismo último de falla de columna fuerte, viga débil, contraviento más débil.

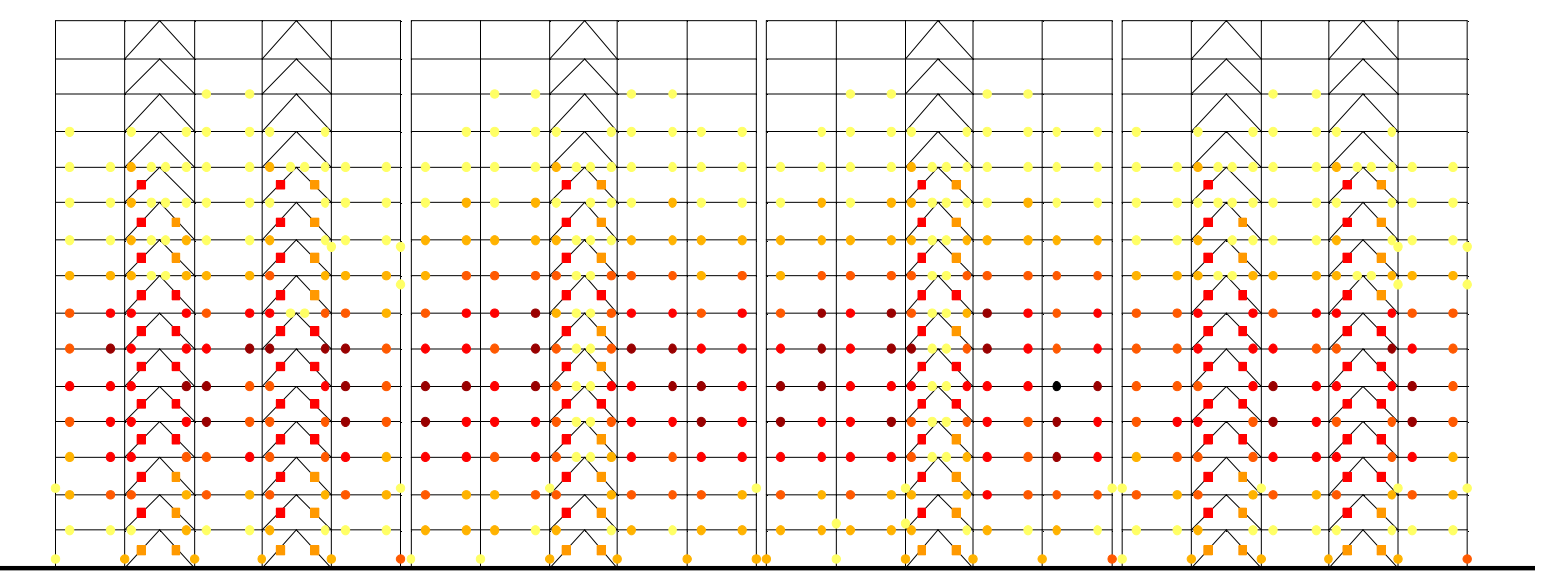

a) Modelo 15XT2 ante el acelerograma 15-EW

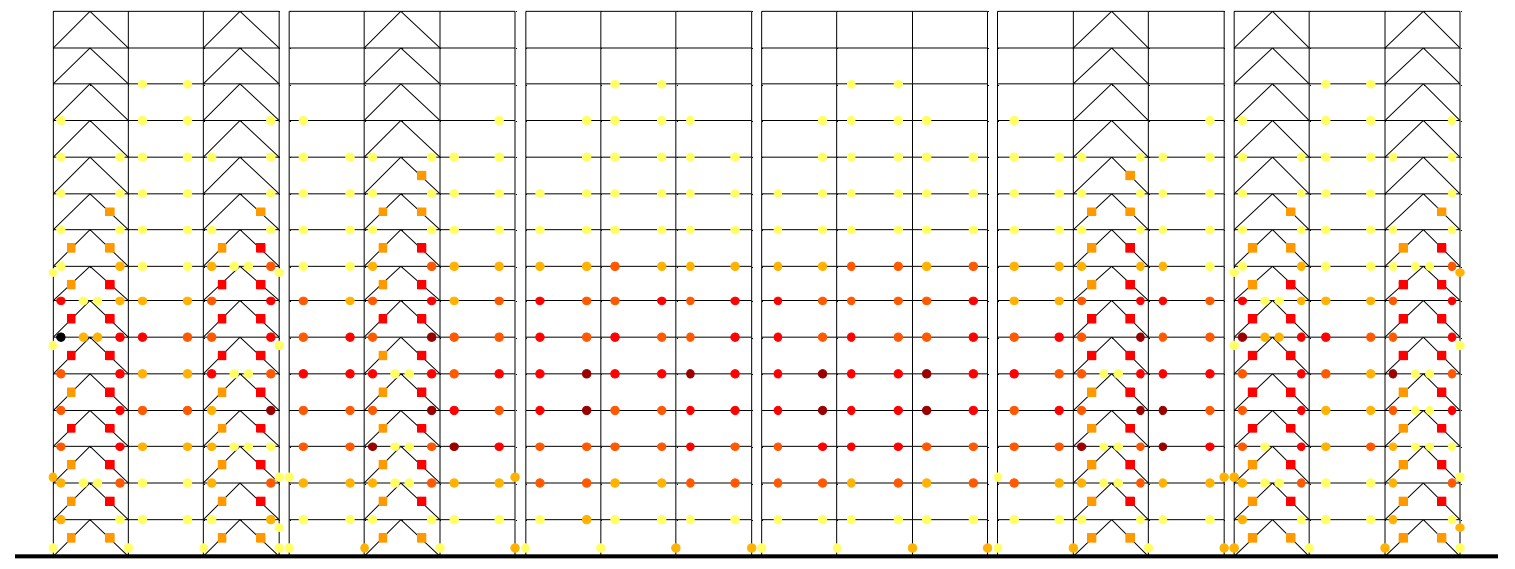

b) Modelo 15 YT2 ante el acelerograma RO-NS

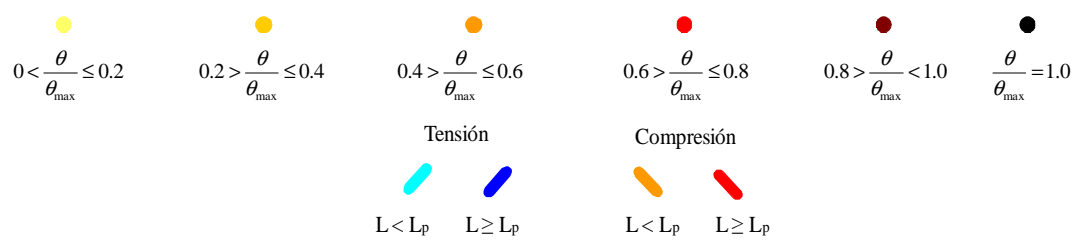

Figura 20. Rotaciones plásticas normalizadas de los modelos correspondientes al edificio tipo 2 ante los acelerogramas que generan las demandas máximas, considerando sobrerresistencia.

De forma complementaria, en las figuras 21 y 22 se muestran respectivamente las respuestas máxima y promedio de los modelos $15 \mathrm{XT} 2$ y $15 \mathrm{YT} 2$. En las figura $21 \mathrm{~b}$ y $22 \mathrm{~b}$ se muestran las distorsiones de fluencia $\left(\Delta_{y}\right)$ de ambos modelos. De los resultados asociados a la respuesta máxima (símbolos llenos), se observan distorsiones mayores en dirección $X$. Sin embargo, al considerar la respuesta promedio (símbolos vacíos), los valores son muy similares en ambas direcciones, pues como se observa de la tabla 2 , las rigideces laterales son muy parecidas en ambas direcciones. Se observa nuevamente que las distorsiones de fluencia concuerdan de manera razonable con el límite propuesto para la revisión del estado límite de servicio $\left(\Delta_{y}=0.002\right)$, lo cual también ocurre en el resto de los modelos. Lo anterior indica 
que también para edificios de esta altura, el límite propuesto para la revisión del estado límite de servicio, con base en los resultados de los análisis estáticos no lineales, es adecuado. De hecho, el promedio de los entrepisos que tienen comportamiento marcadamente inelástico, exceptuando el primero por la condición impuesta de frontera de base rígida, es $\Delta_{y p r o m}=0.0021,0.0020,0.0019$ y 0.0019 para los modelos $15 X T 1$, $15 \mathrm{YT} 1,15 \mathrm{XT} 2$ y $15 \mathrm{YT} 2$, respectivamente.
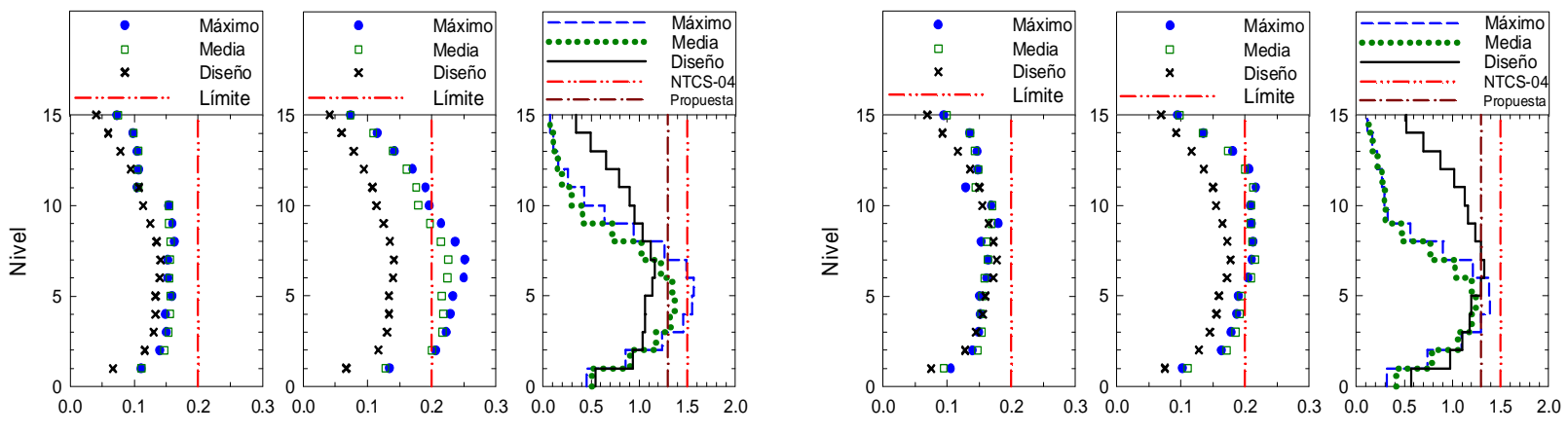

a) Distorsión, $\Delta_{\mathrm{fe}}(\%)$

b) Distorsión, $\Delta_{y}(\%)$

c) Distorsión, $\Delta_{\max }(\%)$
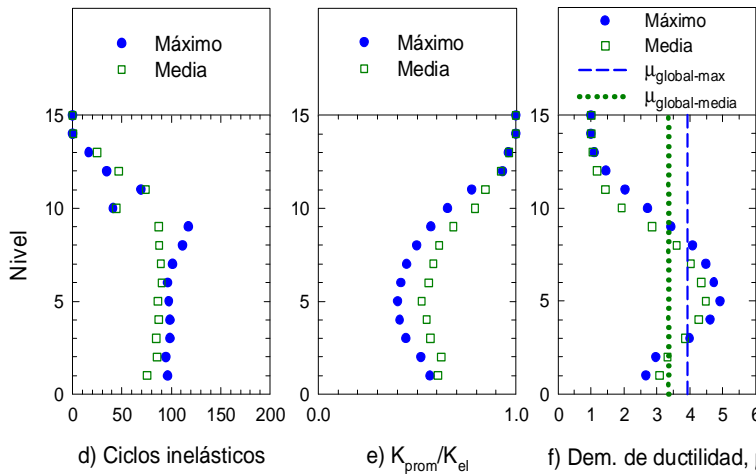

a) Distorsión, $\Delta_{\mathrm{fe}}(\%)$

b) Distorsión, $\Delta_{y}(\%)$

c) Distorsión, $\Delta_{\max }(\%)$
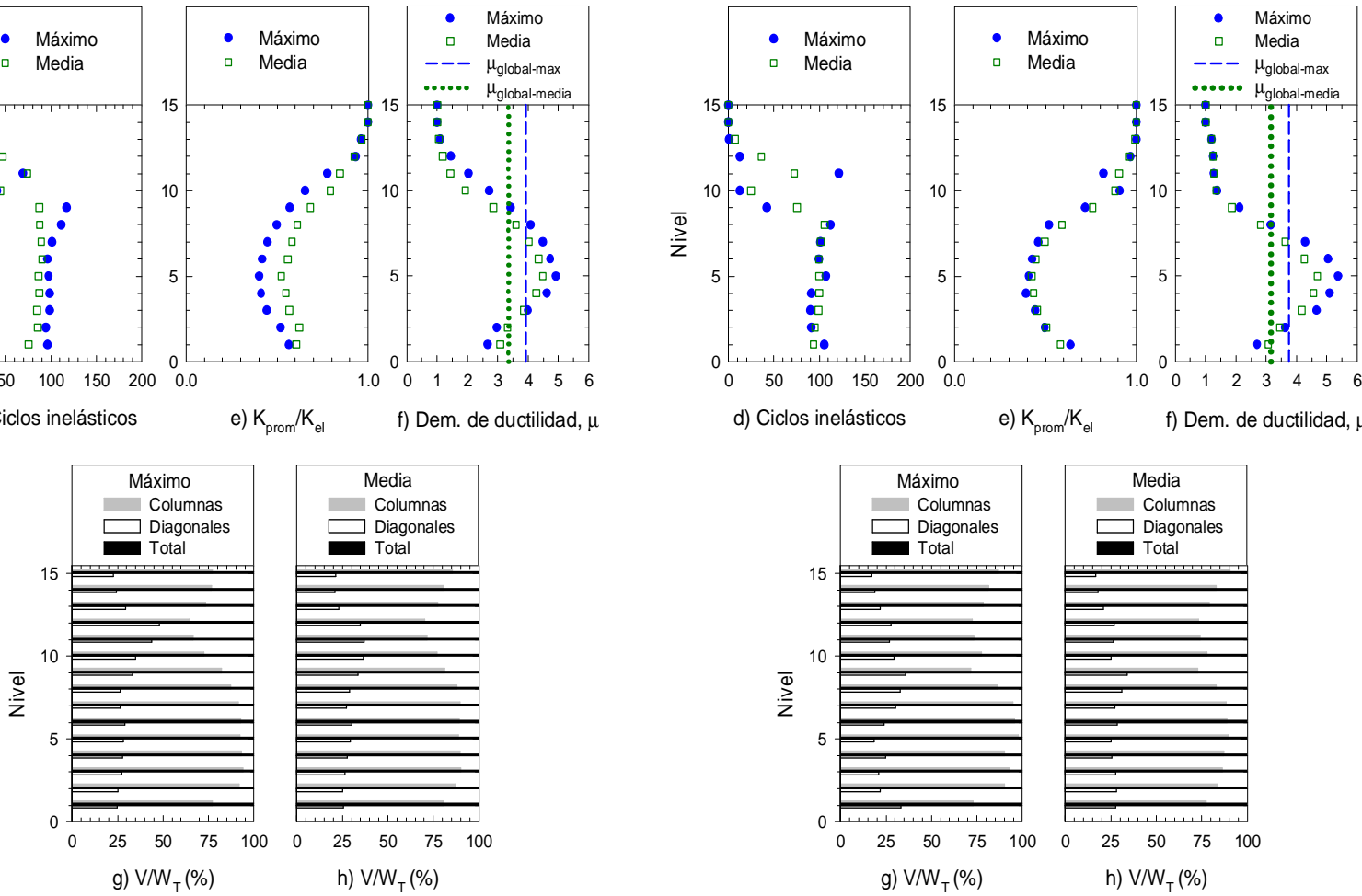

Figura 21. Respuesta máxima y promedio del modelo 15XT2 considerando sobrerresistencia
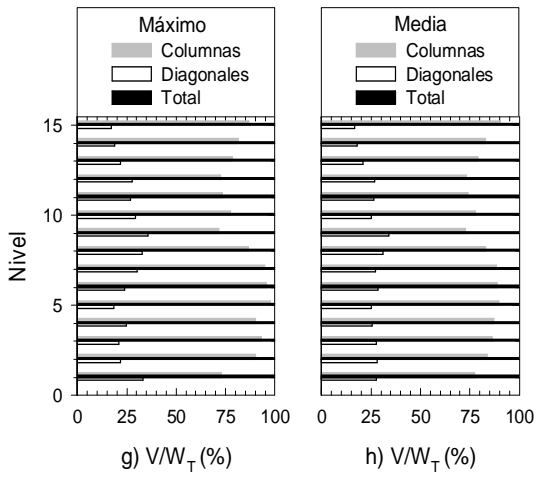

Figura 22. Respuesta máxima y promedio del modelo 15YT2 considerando sobrerresistencia

De las envolventes de respuesta máxima obtenidas para el modelo 15XT2 (línea discontinua, figura 21c), se observan que las demandas de distorsiones de entrepiso son superiores a las observadas en dirección $Y$ (figura 22c). Al igual que en los modelos tipo 1, en dirección $X$, la distorsión en algunos entrepisos ubicados en la altura media exceden el límite propuesto en las NTCS-04 ( $\Delta=0.015)$, en tanto en la dirección $Y$, este valor no se excede en ningún entrepiso. La envolvente de diseño (línea continua) asociada a la revisión del estado límite de seguridad contra colapso cubre adecuadamente las distorsiones 
asociadas a la respuesta máxima en los entrepisos inferiores y superiores; sin embargo, en los entrepisos ubicados en la altura media, la envolvente de diseño no cubre dichas distorsiones (figuras 21c y 22c).

Con base en las respuestas promedio (líneas punteadas), se observaron envolventes de distorsiones muy similares en forma y magnitudes en ambas direcciones, siendo ligeramente superiores las obtenidas en dirección $X$ (figuras 21c y 22c). En ambas direcciones, las distorsiones pico presentaron valores muy cercanos al límite propuesto en esta investigación $(\Delta=0.013)$. De la figura $21 \mathrm{c}$ se observa que las distorsiones de los entrepisos tres a seis exceden los valores definidos por la envolvente de diseño.

De la figura $21 \mathrm{~d}$ se observa, con base en la respuesta promedio (símbolos vacíos), que la demanda de ciclos inelásticos en el modelo $15 \mathrm{XT} 2$ es prácticamente uniforme en los entrepisos dos a nueve. Asimismo, de la figura 20e se observan niveles de degradación de rigidez promedio en todos los entrepisos mayores a 0.5 . Lo cual indica una degradación de rigidez importante en algunos entrepisos.

En las figuras $21 \mathrm{~d}$ y $22 \mathrm{~d}$ se observa, con base en la respuesta promedio (símbolos vacios), que la demanda de ciclos inelásticos es prácticamente uniforme en los entrepisos dos a nueve en el caso del modelo 15XT2, en tanto que para el modelo 15YT2, de los entrepisos dos a ocho. De la respuesta máxima (símbolos llenos), se observaron niveles de degradación de rigidez $\left(K_{p r o m} / K_{e l}\right)$ muy similares en ambas direcciones. Sin embargo, con base en la respuesta promedio (símbolos vacios), congruente con el número de ciclos inelásticos observado, se obtuvieron niveles de degradación de rigidez ligeramente mayores en dirección $Y$ (figura 22e), con valores mínimos cercanos a 0.45 , en tanto en dirección $X$, en todos los entrepisos se tienen valores mayores a 0.5. Lo anterior indica una degradación de rigidez importante en algunos entrepisos, principalmente en dirección $Y$ (entrepisos tres a siete).

Adicionalmente, en las figuras $21 \mathrm{f}$ y $22 \mathrm{f}$ se muestran las envolventes máximas y promedio de demandas de ductilidad de entrepiso, así como las ductilidades globales. De las envolventes de respuestas máximas (símbolos llenos), se observan valores pico cercanos a cinco en los entrepisos cuatro a seis. Se observa además que la demanda de ductilidad global promedio en dirección $X \quad\left(\mu_{\text {global-media }}=3.35\right)$ es superior a la obtenida en dirección $Y\left(\mu_{\text {global-media }}=3.15\right)$. Nuevamente se observaron para las dos direcciones, tanto niveles de degradación de rigidez, como demandas de ductilidad mayores al considerar las envolventes de respuesta máxima respecto a las obtenidas con base en las respuestas promedio.

El análisis conjunto de los tres parámetros comentados (número de ciclos inelásticos, degradación de rigidez, y demandas de ductilidad) indica, al igual que en los modelos tipo 1, que existen tanto ciclos de comportamiento inelástico de amplitud pequeña como grande, pues los niveles de degradación de rigidez y las demandas de ductilidad en algunos entrepisos son elevados. Esto puede corroborarse a partir de la observación de las respectivas curvas de histéresis de donde se extrajo esta información (figura 19), ejemplificando la valía de los tres parámetros reportados para realizar análisis más detallados donde se considere la respuesta promedio de un cierto número de simulaciones, donde sería poco práctico presentar siempre las respectivas curvas de histéresis definidas para cada entrepiso y cada movimiento del terreno.

Respecto a los cortantes de entrepiso máximos en columnas y contravientos $\left(\mathrm{V} / \mathrm{W}_{\mathrm{T}}\right.$, figuras $21 \mathrm{~g}$ y 22g), para los modelos tipo 2, se observaron aportes del sistema de contraventeo ligeramente inferiores que los observados en los modelos tipo 1. En la dirección $X$ se obtuvo un valor medio de $29 \%$, en tanto que para la dirección $Y$ un valor medio de $26 \%$. Lo anterior se debe a que, a pesar de que los modelos tipo 2 cuentan con un número mayor de marcos contraventeados, las secciones empleadas en contravientos en este edificio son más esbeltas que las consideradas en los modelos del edificio tipo 1. En todos los casos, se obtuvieron valores congruentes con los inicialmente planteados en la etapa de diseño. Por ejemplo, para 
el caso de los modelos del edificio tipo 1, se estimó que las columnas aportarían aproximadamente el $65 \%$ de la resistencia a cortante de entrepiso en dirección $X$ y, el 75\% en dirección $Y$.

Como se comentó, las incertidumbres existentes en la determinación del aporte final que a la resistencia a fuerza cortante brinda el sistema de contraventeo y los marcos son de esperarse. Cabe señalar que en la etapa de diseño se determinan las resistencias últimas de marcos y contravientos con base en el cálculo de resistencias nominales, es decir, no se toman en cuenta las resistencias adicionales que pueden desarrollarse por el confinamiento del núcleo del concreto en columnas, del aporte del refuerzo de la losa en vigas y por un esfuerzo de fluencia mayor al nominal en el caso de los contravientos, por ejemplo. Esto explica estas diferencias, en congruencia con los resultados de los análisis estáticos no lineales, donde también se observó una diferencia entre el aporte final predicho y el calculado. Sin embargo, como se observó en la mayoría de los casos, la variación entre los valores predichos y los calculados es pequeña y muy razonable, por lo que no se justificaría, para fines prácticos, complicar la etapa de prediseño, exigiendo cuantificar las sobrerresistencias adicionales por los conceptos antes mencionados.

\section{Modelos de 24 niveles}

En la figura 23 se muestran las curvas de histéresis de los modelos 24XT1 y 24XT2. Como se observa, tanto de las curvas de histéresis, como de los mapeos de rotaciones plásticas normalizadas (figura 24), el mayor comportamiento inelástico en ambos modelos se presenta en los entrepisos ubicados en la parte media baja (entrepisos tres a 12) concentrándose mayoritariamente en las vigas y contravientos. Se observa que la mayoría de los contravientos ubicados en la zona mencionada trabajan inelásticamente; sin embargo, no todos los elementos pandean. Congruente con las curvas de histéresis, en los mapeos de rotaciones plásticas se observa que las vigas del segundo al $12^{\circ}$ entrepiso son las que mayor demanda de trabajó inelástico presentan.
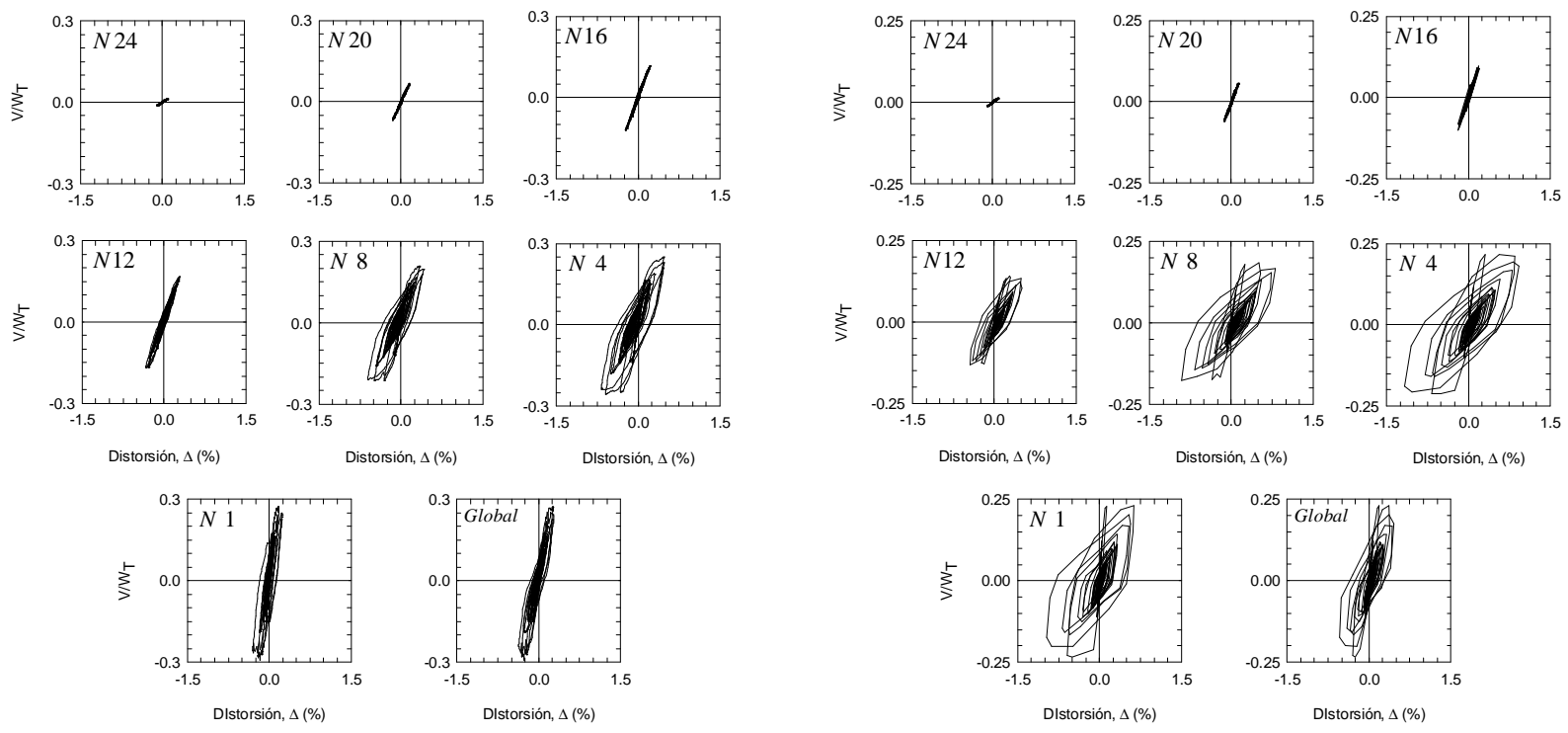

a) Modelo 24XT1
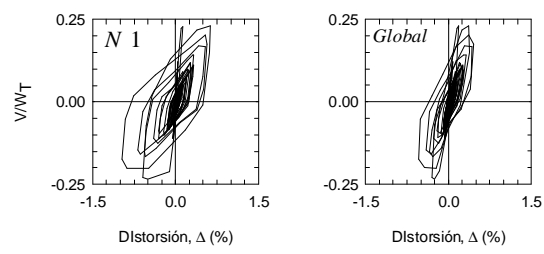

b) Modelo 24XT2

Figura 23. Curvas de histéresis de los modelo 24XT1 y 24XT2 ante los acelerogramas SCT-EW y 04-NS, respectivamente, que generan las demandas máximas, considerando sobrerresistencia. 
Desempeño sísmico de edificios con base en marcos dúctiles de concreto reforzado con contraventeo chevrón

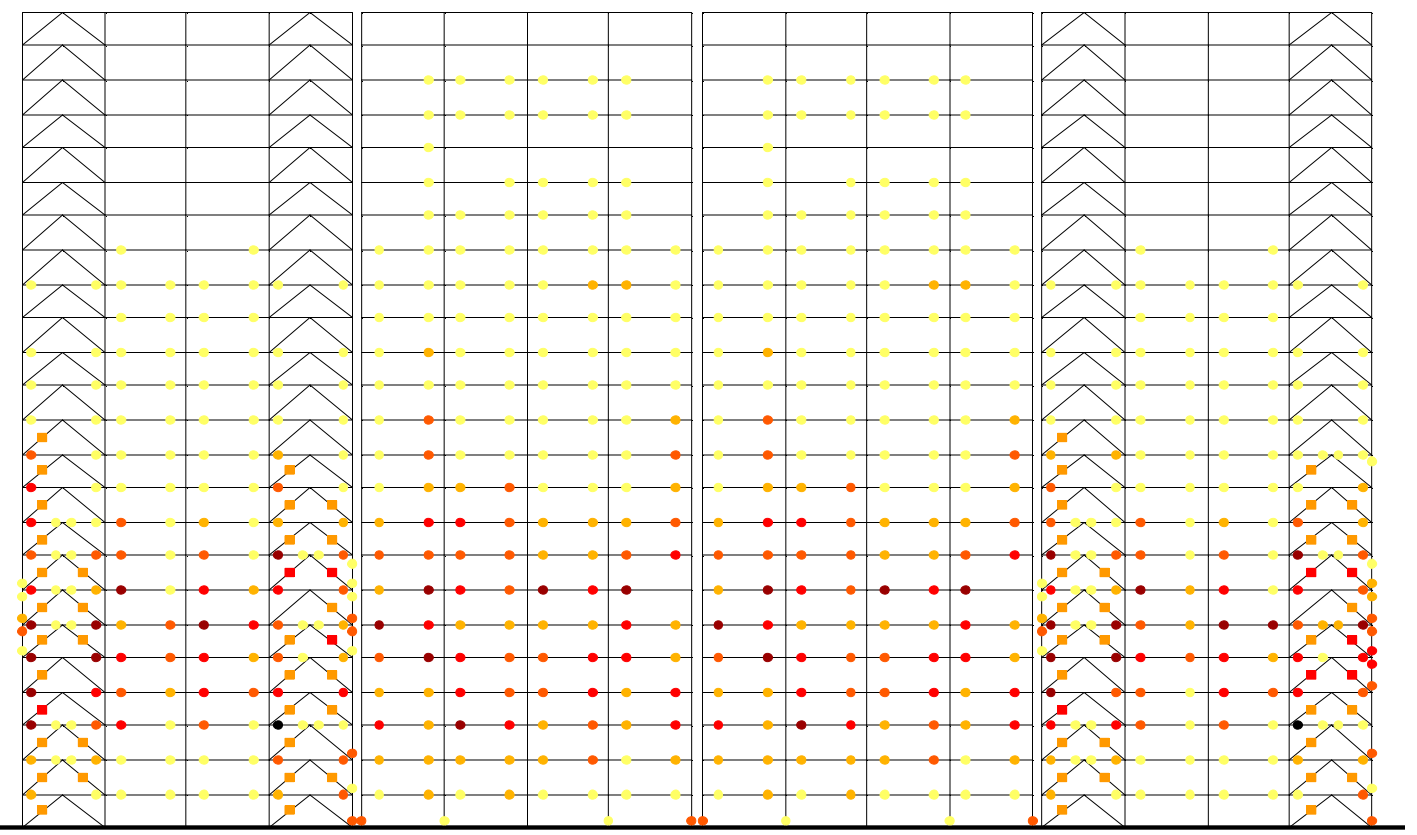

a) Modelo 24XT1 ante el acelerograma SCT-EW

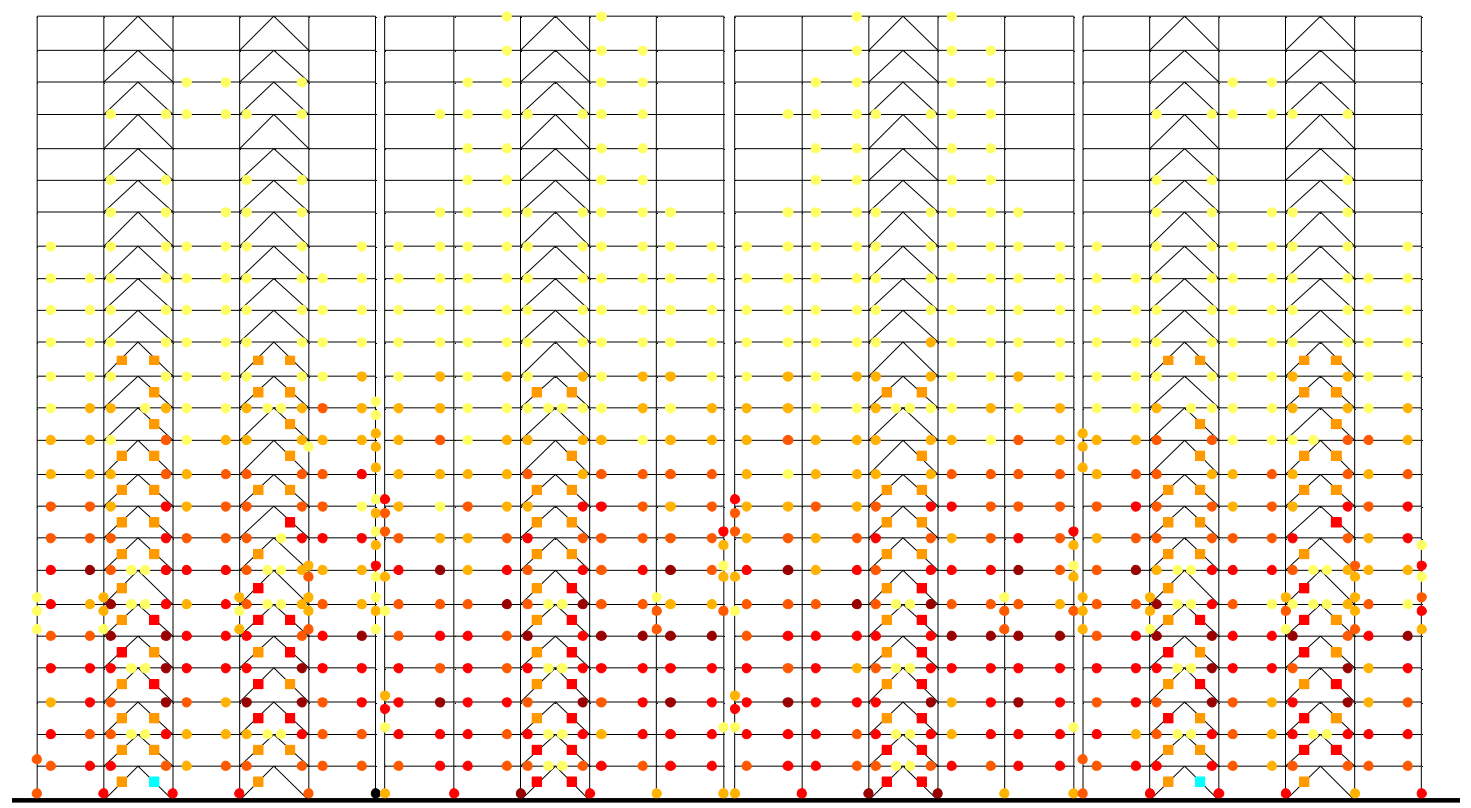

b) Modelo 24XT2 ante el acelerograma 04-NS

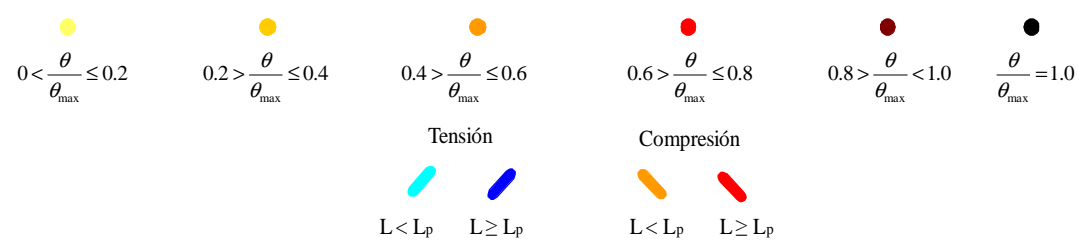

Figura 24. Rotaciones plásticas normalizadas de los modelos 24XT1 y 24 XT2 ante los acelerogramas que generan las demandas máximas, considerando sobrerresistencia. 
De los mapeos de fluencias de ambos modelos (figura 24), se observaron fluencias en la base de las columnas de planta baja, lo cual es aceptable dadas las demandas de distorsión impuestas por los movimientos del terreno y a la condición de empotramiento perfecto considerada en los análisis. Asimismo, se observan algunas fluencias en las columnas de borde de los marcos contraventeados, con rotaciones de magnitud mucho más importantes que las registradas en los modelos de menor altura. Estas fluencias en columnas (columnas del tercer, sexto y séptimo entrepiso) están relacionadas en parte con el cambio en el refuerzo longitudinal en las columnas, que se realiza en el sexto entrepiso, así como con el cambio de secciones transversales que ocurre en el mismo entrepiso.

De lo comentado, se observa que en la mayoría de los casos, las distorsiones de fluencia promedio concuerdan de manera razonable con el límite propuesto para la revisión del estado límite de servicio $\left(\Delta_{y}=0.002\right)$, lo que indica, al igual que en los modelos de ocho y 15 niveles, que el límite propuesto con base en los resultados de los análisis estáticos no lineales para la revisión del estado límite de servicio es adecuado.

Finalmente, se puede concluir, con base principalmente en los resultados derivados de los modelos en dirección $X$, en que se presenta una demanda inelástica mayor a la obtenida en los modelos en dirección $Y$, que los mapeos de rotaciones y deformaciones plásticas muestran que para esta altura, no se satisface completamente la premisa de diseño de mecanismo último de falla de columna fuerte, viga débil, contraviento más débil, pues se observan rotaciones inelásticas en algunas columnas ubicadas en los entrepisos intermedios, lo cual no es deseable para obtener un comportamiento adecuado. Asimismo, no se observa un trabajo inelástico uniforme de las diagonales de contraventeo en la altura, permaneciendo dichos elementos elásticos en los entrepisos ubicados en el tercio superior. De hecho, en el tercio superior las vigas resultaron ser, en general, más débiles que los contravientos, al fluir incluso en los marcos donde se ubican los contravientos, aunque cabe destacar que las magnitudes de estas fluencias son reducidas.

\section{SOBRERRESISTENCIA MÁXIMA DEMANDADA POR LOS MOVIMIENTOS DEL TERRENO}

En esta sección se presentan los niveles de sobrerresistencia inducidos por cada movimiento del terreno empleado. La sobrerresistencia se estima como el cociente entre el cortante máximo normalizado respecto al peso total de cada modelo $\left(V / W_{T}\right)$ respecto al cortante sísmico de diseño $\left(V_{\text {dis }}\right)$. En la figura 25 se hace una comparativa entre los niveles de sobrerresistencia inducidos por cada acelerograma y los factores de reducción por sobrerresistencia $(R)$ empleados para el diseño de cada uno de los edificios. Los factores de reducción por sobrerresistencia de diseño corresponden a la propuesta realizada con base en los resultados de los análisis estáticos no lineales. Para cada modelo se muestran los valores mínimos, máximos y los promedios.

De la figura 25 se observa que, para el intervalo de periodos estudiado, los niveles de sobrerresistencia obtenidos con base en las respuestas máximas y promedios coinciden de buena forma con la propuesta realizada para la estimación de los factores de reducción por sobrerresistencia, para todos los modelos. Las demandas de sobrerresistencia en los modelos de ocho niveles (modelos más rígidos) son ligeramente inferiores a lo observado en el resto de los modelos. Lo anterior se debe a que en estos modelos, como se observó de las curvas de histéresis correspondientes, no existió una demanda inelástica importante, por lo cual las demandas de resistencia no fueron elevadas. De haber existido una demanda inelástica mayor en los modelos de los edificios de ocho niveles, la demanda de fuerza cortante en cada caso podría haberse incrementado y, por ende la sobrerresistencia desarrollada sería un poco mayor. 


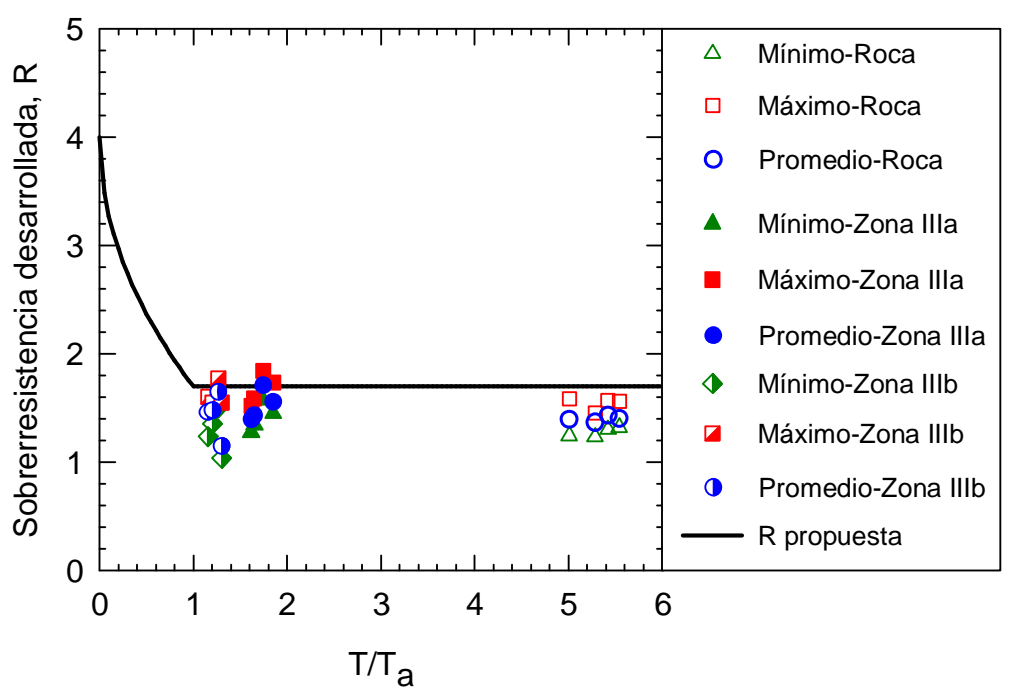

Figura 25. Sobrerresistencias desarrolladas ante los movimientos del terreno

\section{CONCLUSIONES}

Del conjunto de resultados, en los que para obtener respuestas promedio se emplearon como mínimo siete registros acelerométricos, se pueden hacer los siguientes comentarios y observaciones:

(1) Con base en las respuestas promedio de todos los edificios (ocho, 15 y 24 niveles) se observa que las distorsiones de fluencia, calculadas con base en un ajuste elasto-plástico, concuerdan de manera razonable con el límite propuesto para la revisión del estado límite de servicio $\left(\Delta_{y}=0.002\right)$, el cual se basa en los resultados obtenidos en la primera etapa de esta investigación. Lo anterior indica que para edificios con relaciones de esbeltez $0.4 \leq H / L \leq 4$, el límite propuesto para la revisión del estado límite de servicio, con base en los resultados de los análisis estáticos no lineales, es adecuado. El parámetro anterior se considera de importancia, ya que actualmente las NTCS-04 establecen $\Delta_{y}=0.004$ para todos los sistemas estructurales y, como se demuestra, ese límite no es adecuado para el sistema estructural en estudio.

(2) De las envolventes máximas y promedio de distorsiones de entrepiso dinámicas, obtenidas principalmente para los modelos de 15 y 24 niveles, que representan los modelos en que se experimentó mayor demanda inelástica, se puede concluir que el límite de distorsión asociado a la revisión del estado límite de colapso actualmente propuesto en las NTCS-04, así como en el ASCE-7-05 $\left(\Delta_{\max }=0.015\right)$, cubre de mejor manera las demandas de distorsión observadas, que el límite propuesto en la primera parte de esta investigación con base en los análisis estáticos no lineales $\left(\Delta_{\max }=0.013\right)$. Los resultados derivados de los análisis dinámicos no lineales paso a paso respaldan con evidencia numérica esta disposición reglamentaria.

(3) Se puede concluir, con base en los mapeos de rotaciones y deformaciones plásticas, que para los edificios de altura baja a mediana (ocho y 15 niveles) se satisface muy razonablemente la premisa de diseño de mecanismo último de falla de columna fuerte, viga débil, contraviento más débil. Sin embargo, los mecanismos de falla asociados a los modelos de 24 niveles no satisfacen por 
completo esta premisa de diseño, pues existen rotaciones plásticas en algunas de las columnas ubicadas en los entrepisos intermedios, con magnitudes significativas, debidas en parte a los cambios de refuerzo longitudinal y cambios de secciones transversales. Debe considerarse que en estos casos, las secciones de diseño eran poco eficientes desde un punto de vista práctico, debido en gran medida a que los edificios se ubicaron en la zona de mayores demandas sísmicas del terreno blando del Distrito Federal.

(4) Se observó en los modelos de 15 y 24 niveles que la rigidez efectiva promedio de algunos entrepisos tiene valores del orden de la mitad de la rigidez elástica inicial, lo cual se considera aceptable para los niveles de distorsiones demandadas por los movimientos del terreno. Los entrepisos en que se observan los mayores niveles de degradación de rigidez concuerdan con aquéllos en que se experimenta un número de ciclos inelásticos importante y están asociadas a demandas de ductilidad de entrepiso superiores a cuatro.

(5) Con base en los valores máximos y promedio de los niveles de sobrerresistencia inducidos por cada movimiento del terreno, se concluye que el factor de reducción por sobrerresistencia $(R$, ec. 1 ), propuesto con base en los análisis estáticos no lineales, es congruente y adecuado para el diseño de estructuras con base en marcos de concreto reforzado con contraventeo metálico tipo chevrón.

El sistema estructural estudiado tiene aplicabilidad para cualquiera de las zonas estudiadas (Costa de Guerrero y Distrito Federal), pues ambas representan zonas de alto peligro sísmico en México. Una de las principales ventajas de este sistema estructural radica en la disminución de la masa reactiva de la estructura en comparación con otros sistemas estructurales para resistir cargas laterales, como lo son los muros de concreto. Asimismo, algunos autores han mostrado que el uso de sistemas de contraventeo con configuración en cruz (X) no es adecuado si se requiere de un comportamiento dúctil (Maheri y Akbari 2003).

Finalmente, de lo observado en las dos partes de esta investigación, se concluye que si se emplea la metodología de diseño por capacidad propuesta en un estudio alterno (Godínez y Tena 2011), tanto a nivel elemento, como global, es factible realizar diseños dúctiles de edificios de baja a mediana altura (menores a 20 pisos) en terrenos blandos de la zona metropolitana de la Ciudad de México en que se obtengan mecanismos cercanos al supuesto de columna fuerte - viga débil - contraviento más débil, capacidades de deformación y de sobrerresistencia razonables para marcos de concreto reforzado contraventeados con perfiles metálicos de baja y mediana altura, cuando las columnas de los marcos contribuyen por lo menos con el $50 \%$ de la resistencia a fuerza cortante del entrepiso. Lo anterior es congruente considerando tanto los resultados de los análisis estáticos no lineales realizados a modelos de marcos, como los resultados de los análisis dinámicos no lineales paso a paso realizados a modelos de edificios más complejos.

Por el contrario, de los resultados derivados tanto de los análisis estáticos no lineales, como de los dinámicos no lineales paso a paso, se observa que los modelos de mayor altura ( 24 niveles) en la zona del lago de la Ciudad de México desarrollan capacidades de deformación inferiores que los modelos de menor altura, debido en gran medida al incremento de la carga axial por sismo en las columnas ubicadas en los primeros entrepisos. Por lo anterior, el obtener diseños dúctiles en dichos casos es una tarea complicada, principalmente al considerar edificios ubicados en zonas donde las ordenadas del espectro de diseño elástico de aceleración son relativamente altas, como fue el caso considerado en este estudio (zona III de las NTCS-04). Sin embargo, lo anterior no indica que para zonas de menor sismicidad y/o con ordenadas espectrales inferiores (por ejemplo, zona I de las NTCS-04), no se puedan realizar diseños con base en el sistema estructural estudiado de edificios con alturas semejante a las comentadas, en que se obtengan 
mecanismos de colapso congruentes con el esperado, columna fuerte-viga débil-contraviento más débil. El corroborar el punto anterior es una tarea pendiente, la cual se realizará en futuras investigaciones.

Debe considerarse que para la aplicación de los resultados derivados de este estudio, las conexiones del sistema de contraventeo metálico al marco de concreto reforzado, deben diseñarse como rígidas; es decir, las componentes de deformación se deberán exclusivamente a la deformación de los elementos estructurales. Asimismo, el comportamiento de la conexión debe ser congruente con el objetivo de diseño, es decir, presentar un comportamiento rígido-elástico, por lo que no debe presentarse fluencia por flexión y aún menos una falla prematura por cortante.

\section{AGRADECIMIENTOS}

El primer autor agradece la beca de Conacyt que le permitió solventar por cuatro años sus estudios e investigación doctorales. Los autores agradecen a la Dirección de Obras del Gobierno del Distrito Federal por el pequeño patrocinio complementario en la fase final de esta investigación. Se agradecen ampliamente los comentarios y sugerencias de los revisores anónimos, los cuales ayudaron a mejorar la calidad de este artículo.

\section{REFERENCIAS}

Black, R, W Wenger y E Popov (1980), "Inelastic buckling of steel struts under cyclic load reversals" Reporte No. UCB/EERC-80/40, Department of Civil Engineering, University of California at Berkeley.

Carr, A J (2004), "RUAUMOKO: computer program library", Department of Civil Engineering, University of Canterbury, Nueva Zelanda.

Correa, H (2005), "Evaluación de los criterios de diseño sísmico de la propuesta de Reglamento de Construcciones para el Distrito Federal (RCDF-2001) para edificios regulares con base en marcos dúctiles de concreto reforzado", Tesis de Maestría, División de Estudios de Posgrado de la Facultad de Ingeniería, Universidad Nacional Autónoma de México, mayo.

ETABS (2005), "ETABS Nonlinear Versión 9.0.0, Extended 3D analysis of building systems", Computer and Structures, Inc., Berkeley, California.

Gatica, G (2007), "Importancia de la sobrerresistencia que aportan las losas a las vigas de edificios con base en marcos dúctiles diseñados conforme a los reglamentos del Distrito Federal", Tesis de Maestría, División de Estudios de Posgrado de la Facultad de Ingeniería, Universidad Nacional Autónoma de México.

Ghaffarzadeh, H y M R Maheri (2006), "Cyclic tests on internally braced frames”, Journal of Seismology and Earthquake Engineering, Vol. 8, No. 3, pp. 177-186.

Godínez, E A (2005), "Evaluación de la vulnerabilidad sísmica de estructuras existentes en el Distrito Federal. El caso específico del sismo del 19 de septiembre de 1985", Tesis de Maestría, Posgrado en Ingeniería Estructural, División de Ciencias Básicas e Ingeniería, Universidad Autónoma Metropolitana Azcapotzalco, mayo.

Godínez, E A, A Tena y L E Pérez (2005), "Vulnerabilidad de estructuras con base en marcos de concreto reforzado en el Valle de México ante un escenario similar al sismo de septiembre de 1985", Revista de Ingeniería Sísmica, SMIS, No. 73, pp. 71-101, julio-diciembre. 
Godínez, E A y A Tena (2007), "Evaluación de los criterios de diseño sísmico del RCDF para marcos dúctiles de concreto reforzado con contravientos", Memorias, XVI Congreso Nacional de Ingeniería Sísmica, Ixtapa, Guerrero, CD-ROM, noviembre.

Godínez-Domínguez, E A y A Tena-Colunga (2008), "Behavior of moment resisting reinforced concrete concentric braced frames (RC-MRCBFS) in seismic zones", Memorias, 14th World Conference on Earthquake Engineering, Beijing, China, Artículo No. 05-03-0059, CD-ROM, octubre.

Godínez, E A y A Tena (2009), "Propuesta de diseño de marcos dúctiles de concreto reforzado con contraventeo chevrón con base en los resultados de análisis no lineales", Memorias, XVII Congreso Nacional de Ingeniería Sísmica, Puebla, Puebla, CD-ROM, noviembre.

Godínez, E A y A Tena (2011), "Comportamiento no lineal de marcos dúctiles de concreto reforzado con contraventeo chevrón. Propuesta de diseño", Revista de Ingeniería Sísmica, SMIS, No. 85, pp. 61102, julio-diciembre.

Godínez-Domínguez, E A y A Tena-Colunga (2010), "Nonlinear behavior of code-designed reinforced concrete concentric braced frames under lateral loading", Engineering Structures, Vol. 32, pp. 944963.

Godínez, E A (2010), "Estudio del comportamiento de marcos dúctiles de concreto reforzado con contraventeo chevrón", Tesis de Doctorado, Posgrado en Ingeniería Estructural, División de Ciencias Básicas e Ingeniería, Universidad Autónoma Metropolitana Azcapotzalco, julio.

Horvilleur, J F y M A Cheema (1994), "Análisis lateral de marcos de concreto reforzado sometidos a cargas laterales sísmicas", Memorias, IX Congreso Nacional de Ingeniería Estructural, Zacatecas, Zacatecas, Vol. I, pp. 244-259, octubre.

Ikeda, K, y S Mahin (1984), "A refined physical theory for predicting the seismic behavior of braced steel frames" Reporte No. UCB/EERC-84/12, Department of Civil Engineering, University of California at Berkeley.

Luaces, F L (1995), "Sobrerresistencia en estructuras a base de marcos de concreto reforzado", Tesis de Maestría, División de Estudios de Posgrado de la Facultad de Ingeniería, Universidad Nacional Autónoma de México, noviembre.

Luna, J L (2000), "Estudio de los criterios del RCDF-93 y sus normas técnicas complementarias para el diseño sísmico de edificios regulares a base de marcos de concreto", Tesis de Maestría, División de Estudios de Posgrado de la Facultad de Ingeniería, Universidad Nacional Autónoma de México, enero.

Maheri, M R y H Sahebi (1997), "Use of steel bracing in reinforced concrete frames", Engineering Structures, Vol. 19, Vol. 12, pp. 1018-1024.

Maheri, M R y R Akbari (2003). "Seismic behaviour factor, R, for steel X-braced and knee-braced RC buildings", Engineering Structures, Vol. 25, pp. 1505-1513.

Maheri, M R, R Kousari y M Razazan (2003). "Pushover tests on steel X-braced and knee-braced RC frames", Engineering Structures, Vol. 25, pp. 1697-1705.

Maheri, M R y A Hadjipour (2003). "Experimental investigation and design of steel brace connection to RC frame", Engineering Structures, Vol. 25, pp. 1707-1714.

Maheri, M R y H Ghaffarzadeh (2008), "Connection overstrength in steel-braced RC frames", Engineering Structures, Vol. 30, pp. 1938-1948. 
Miranda, E y J Ruiz (2002), "Influence of stiffness degradation on strength demands of structures built on soft soil sites", Engineering Structures, Vol. 24, pp. 1271-1281.

MOC-2008 (2009), "Manual de diseño de obras civiles. Diseño por sismo", Comisión Federal de Electricidad, noviembre.

NTCC-04 (2004), "Normas Técnicas Complementarias para Diseño de Estructuras de Concreto", Gaceta Oficial del Distrito Federal, Tomo II, No. 103-BIS, octubre.

NTCS-04 (2004), "Normas Técnicas Complementarias para Diseño por Sismo", Gaceta Oficial del Distrito Federal, Tomo II, No. 103-BIS, octubre.

Pérez-Rocha, L E (1998), "Respuesta sísmica estructural: efectos de sitio e interacción suelo-estructura (aplicaciones al valle de México)", Tesis de Doctorado, División de Estudios de Posgrado de la Facultad de Ingeniería, Universidad Nacional Autónoma de México.

RCDF-04 (2004), "Reglamento de Construcciones del Departamento del Distrito Federal”, Gaceta Oficial del Distrito Federal, octubre.

Remennikov, A y W Walpole (1997), "Analytical prediction of seismic behaviour for concentricallybraced steel systems", Earthquake Engineering and Structural Dynamics, Vol. 26, pp. 859-874.

Tena, A, J L Luna, E Filloy y E Del Valle (1997), "Evaluación de las recomendaciones del RCDF para el diseño por sismo de estructuras con marcos de concreto reforzado", Reporte FJBS/CIS-97/04, Centro de Investigación Sísmica, AC, Fundación Javier Barros Sierra, octubre.

Tena, A (2007), Análisis de estructuras con métodos matriciales, primera edición, editorial Limusa, septiembre.

Tena-Colunga, A, E A Godínez-Domínguez y L E Pérez-Rocha (2007), "Vulnerability maps for reinforced concrete structures for Mexico City's Metropolitan Area under a design earthquake scenario", Earthquake Spectra, Vol. 23, No. 4, pp. 809-840.

Tena, A y H Correa (2008), "Evaluación de los criterios de diseño por sismo del RCDF para marcos dúctiles de concreto reforzado", Revista de Ingeniería Sísmica, SMIS, No. 78, pp. 79-101, enerojunio.

Tena-Colunga, A, H Correa-Arizmendi, J Luna-Arroyo y G Gatica-Avilés (2008), "Seismic behavior of code-design medium rise special moment-resisting frame RC buildings in soft soils of Mexico City", Engineering Structures, Vol. 30, No. 12, pp. 3681-3707, doi:10.1016/j.engstruct.2008.05.026.

Tena-Colunga, A, U Mena-Hernández, L E Pérez-Rocha, J Avilés, M Ordaz y J I Vilar (2009), "Updated seismic design guidelines for buildings of a model code of Mexico", Earthquake Spectra, Vol. 25, No. 4, pp. 869-898, noviembre, doi: 10.1193/1.3240413.

Tena-Colunga, A (2010), "Review of the soft first story irregularity condition of buildings for seismic design" The Open Civil Engineering Journal, Vol. 4, pp. 1-15.

Terán, A (2005), "Consideraciones para establecer la resistencia lateral de diseño de estructuras ubicadas en la zona del lago del D.F.”, Memorias, XV Congreso Nacional de Ingeniería Sísmica, México, DF, CDROM, documento No. VI-01, septiembre.

Youssef, M A, H Ghaffarzadeh y M Nehdi (2007). "Seismic performance of RC frames with concentric internal steel bracing”, Engineering Structures, Vol. 29, pp. 1561-1568. 